\title{
Time course of homeostatic structural plasticity in response to optogenetic stimulation in mouse anterior cingulate cortex
}

\author{
Abbreviated title: Homeostatic plasticity induced by stimulation
}

Han $\mathrm{Lu}^{1,2}$, Júlia V. Gallinaro ${ }^{1,3}$, Claus Normann ${ }^{4,5}$, Stefan Rotter ${ }^{1}$, Ipek Yalcin $^{2,6}$

${ }^{1}$ Bernstein Center Freiburg \& Faculty of Biology, University of Freiburg, 79104 Freiburg, Germany

${ }^{2}$ Centre National de la Recherche Scientifique, Université de Strasbourg, Institut des Neurosciences Cellulaires et Intégratives UPR3212, 67000 Strasbourg, France

${ }^{3}$ Bioengineering Department, Imperial College London, London SW7 2AZ, United Kingdom

${ }^{4}$ Department of Psychiatry and Psychotherapy, Medical Center, University of Freiburg

- Faculty of Medicine, University of Freiburg, 79104 Freiburg, Germany

${ }^{5}$ Center for Basics in Neuromodulation, 79104 Freiburg, Germany

${ }^{6}$ Department of Psychiatry and Neuroscience, Université Laval, Québec QC G1J 2G3, Canada

Shared corresponding authors:

Stefan Rotter

Bernstein Center Freiburg \& Faculty of Biology, University of Freiburg, 79104 Freiburg, Germany

Hansastraße 9a, 79104 Freiburg, Germany; stefan.rotter@bio.uni-freiburg.de Ipek Yalcin

Centre National de la Recherche Scientifique, Université de Strasbourg, Institut des Neurosciences Cellulaires et Intégratives UPR3212, 67000 Strasbourg, France

8 Allée du Général Rouvillois, 67000 Strasbourg, France; yalcin@inci-cnrs.unistra.fr

Key words: ACC, synaptic protein, dendritic morphology, depressive disorder, network remodeling, spiking network model 
Number of pages: 78 pages (including 24 pages of Supplementary Materials and Extended Data)

Number of figures: 17 (including 9 supplementary figures)

Number of multimedia: 0

Number of 3D models: 0

Number of words for abstract: 250 words

Number of words for significance statement: 109 words

Number of words for introduction: 619 words

Number of words for discussion: 1476 words

Conflict of interests: The authors declare no competing financial interests.

\section{Acknowledgements:}

We thank Elisabeth Waltisperger and Alessandro Bilella for advice on immunohistochemical staining. We thank Stephan Doridot and the Chronobiotron animal facilities for breeding and genotyping the mice. We thank Beyza Ayazgok for the efforts in molecular analysis. We thank Josef Bischofberger, Stefan Vestring, and Cyril Bories for their help setting up the microinjection experiments. We also thank the staff of the Life Imaging Center (LIC) in the Center for Biological Systems Analysis (ZBSA) of Freiburg University, in particular A. Naumann and R. Nitschke, for help with their confocal microscopy resources and their excellent support in image recording and analysis. Advice and code review by Martin Mörsdorf concerning the statistical analysis using linear mixed models are sincerely acknowledged. The authors also thank Sylvain Hugel, Rudi Tong, and Sandra Diaz-Pier for useful discussions. We also thank Uwe Grauer from the Bernstein Center Freiburg, as well as Bernd Wiebelt and Michael Janczyk from the Freiburg University Computing Center for their assistance with HPC applications and data storage.

This work was funded by the Universitätsklinikum Freiburg, University of Strasbourg, Centre National de la Recherche Scientifique (Grant/Award Number: UPR3212), NARSAD Young Investigator Grant from the Brain \& Behavior Research Foundation (Grant/Award Number: 24736) and Young Talent Award from the University of Stras- 
bourg (IdEx award). Additional funding was obtained by BrainLinks-BrainTools (funded by the Federal Ministry of Economics, Science and Arts of Baden-Württemberg within the sustainability program for projects of the excellence initiative II) and by the Carl Zeiss Stiftung. We are grateful for support by the state of Baden-Württemberg through bwHPC and the German Research Foundation (DFG) through grant no INST 39/963-1 FUGG (bwForCluster NEMO). HL is funded by a NEUREX fellowship and the DeutschFranzösische Hochschule. 


\begin{abstract}
Synapse formation and network rewiring is key to build neural circuits during development and has been widely observed in adult brains. Maintaining neural activity with the help of synaptic plasticity is essential to enable normal brain function. The model of homeostatic structural plasticity (HSP) was proposed to reflect the homeostatic regulation of neural activity and explain structural changes seen after perturbations. However, the specific temporal profile of such plastic responses has not yet been elucidated in experiments. To address this issue, we combined computational modeling and mouse optogenetic stimulation experiments. Our model predicted that within $48 \mathrm{~h}$ post-stimulation, neural activity returns to baseline, while the connectivity among stimulated neurons follows a very specific transient increase and decrease. To capture such dynamics experimentally in vivo, we activated the pyramidal neurons in the anterior cingulate cortex of mice and harvested their brains at $1.5 \mathrm{~h}, 24 \mathrm{~h}$, and $48 \mathrm{~h}$ post-stimulation. Cortical hyperactivity as demonstrated by robust c-Fos expression persisted up to $1.5 \mathrm{~h}$ and decayed to baseline after $24 \mathrm{~h}$. However, spine density and spine head volume were increased at $24 \mathrm{~h}$ and decreased at 48 h. Synaptic proteins VGLUT1 and PSD-95 were also upregulated and downregulated at $24 \mathrm{~h}$ and $48 \mathrm{~h}$, respectively, while the calmodulin-binding protein neurogranin was translocated from the soma to the dendrite. Additionally, lasting astrocyte reactivation and microglia proliferation were observed, suggesting a role of neuron-glia interaction. All this corroborates the interpretation of our experimental results in terms of homeostatic structural plasticity. Our results bring important insights of how external stimulation modulates synaptic plasticity and behaviors.
\end{abstract}




\section{Significance Statement}

We combined both computational modeling and mouse experiments to clarify the temporal dynamics of structural and functional homeostatic plasticity in response to external stimulation. We observed the biphasic regulation of spine density, spine head volume, and synaptic proteins at $24 \mathrm{~h}$ and $48 \mathrm{~h}$ after the optogenetic stimulation of the anterior cingulate cortex, when the neural activity was restored to the homeostatic level. The orchestrated regulation of presynaptic VGLUT1 and postsynaptic PSD-95, as well as the soma-dendrites translocation of neurogranin, suggested an elaborate molecular mechanism underlying homeostatic structural plasticity. Our experimental results thus corroborated the theoretical concept of homeostatic structural plasticity and revealed the temporal evolution of structural and functional plasticity. 


\section{Introduction}

Neural circuits in the mammalian brain are highly plastic. This plasticity is the mechanistic basis of network assembly during development, of use-dependent adaptation and learning in the adolescent and adult brain, but also of decay during ageing and disease (Lamprecht and LeDoux, 2004). In functional plasticity, chemical synapses change their strength by modifying how neurotransmitters and receptors accomplish synaptic signal transmission. Structural plasticity refers to more profound changes, where entirely new synaptic contacts are formed and existing contacts are pruned (Trachtenberg et al., 2002; Caroni et al., 2012; Pfeiffer et al., 2018). Structural plasticity, such as changes in spine volume and spine number, has been shown to accompany and support functional plasticity (Engert and Bonhoeffer, 1999; Matsuzaki et al., 2004; Zhou et al., 2004).

Although structural changes occur spontaneously under baseline conditions, experience significantly accelerates the turnover and induces systematic substantial changes of synaptic connectivity (Holtmaat and Svoboda, 2009). Examples are learning-induced axon sprouting in the hippocampus (Ramırez-Amaya et al., 2001), reorganization of dendritic arbors upon deafferentation in sensory cortices (Oray et al., 2004), induction of synaptogenesis after repetitive glutamate application (Tominaga-Yoshino et al., 2008), and changes of spine morphology in response to disease, injury or stimulation (Knott et al., 2002; Brown et al., 2009; Lai et al., 2012). Such experience-dependent regulation follows two major directions (Fauth and Tetzlaff, 2016). Hebbian plasticity depends on the correlation of neuronal activity, often summarized by "fire together, wire together" (Lowel and Singer, 1992). Homeostatic plasticity, in contrast, counteracts the external perturbation to stabilize activity, employing negative feedback control (Turrigiano, 2012). Robust firing rate homeostasis has been demonstrated for visual cortex neurons during circadian light-dark cycles (Hengen et al., 2016; Pacheco et al., 2019). As the homeostatic regulation is slow, it does not compromise fast information processing (Ma et al., 2019).

The concept of homeostatic structural plasticity (HSP) reconciles structural plasticity with the idea of homeostatic regulation (Butz et al., 2009; Van Ooyen, 2011; Butz and 
van Ooyen, 2013). According to this rule, a neuron creates new dendritic spines and new axonal boutons when its level of activity is below a set-point, and it decreases the number of spines and boutons when its activity exceeds this set-point. The formation of spines and boutons depends exclusively on the neuron's own activity level, and synapses are formed by randomly merging the new spines and boutons. It was recently shown in computer simulations that homeostatic structural plasticity in spiking neural networks typically has a biphasic temporal profile and exhibits associative Hebbian-like properties (Gallinaro and Rotter, 2018). In experiments, transcranial direct current stimulation (tDCS) (Reato et al., 2013) and optogenetic stimulation (Cardin et al., 2010; Barthas et al., 2015) are often employed to perturb neural network activity and induce synaptic plasticity. We showed in another recent simulation study that the long-lasting after-effects of tDCS could be explained by homeostatic structural plasticity (Lu et al., 2019). In our present experimental study, we evaluated the time course of synaptic connectivity changes driven by optogenetic stimulation in mice.

For this purpose, we combined computational modeling and mouse optogenetic experiments. We predicted the temporal response profiles of excitatory neurons using a neural network model implementing homeostatic structural plasticity. An in vivo mouse model was then adopted to measure changes of neural activity and connectivity upon optogenetic stimulation in the anterior cingulate cortex (ACC). The time course of changes was sampled at $1.5 \mathrm{~h}, 24 \mathrm{~h}$ and $48 \mathrm{~h}$ post-stimulation. We analyzed the ACC pyramidal neurons morphology as well as the relative abundance and localization of certain synaptic proteins such as the vesicular glutamate transporter (VGLUT1), the postsynaptic density scaffold protein PSD-95, which show activity-dependent regulation (De Gois et al., 2005; Ehrlich et al., 2007) and another postsynaptic protein, neurogranin (Prichard et al., 1999).

We used repetitive optogenetic stimulation to perturb the activity of pyramidal neurons, evidenced by a robust c-Fos expression at $1.5 \mathrm{~h}$. When neural activity had finally returned to baseline $24 \mathrm{~h}$ after the end of the stimulation, dendritic spine density and spine head volume of the stimulated mice were increased, as predicted by the computational model. After $48 \mathrm{~h}$, both were back to their previous levels, or even slightly below 
them. Histochemical analysis confirmed that VGLUT1 and PSD-95 were upregulated after $24 \mathrm{~h}$ and then again downregulated after $48 \mathrm{~h}$. The expression of neurogranin was unaltered, but it was translocated from the soma to the dendrites of the same neuron. We also found that glial markers, GFAP and IBA1 were overexpressed throughout the period of $48 \mathrm{~h}$ after stimulation, suggesting that glial activity was involved in homeostatic regulation. 


\section{Materials and Methods}

\section{Neuron, synapse, and network models}

Numerical simulations of networks with homeostatic structural plasticity were used as a framework to interpret the outcome of our various measurements in mouse experiments. We used the same neuron model, synapse model, and network architecture, as published in our previous paper on transcranial electric stimulation (Lu et al., 2019). All the plastic neuronal network simulations of the current study were performed with the NEST simulator using a MPI-based parallel configuration (Linssen et al., 2018).

The current-based leaky integrate-and-fire (LIF) neuron model was used for both excitatory and inhibitory neurons. We employed an inhibition-dominated recurrent network with 10000 excitatory and 2500 inhibitory neurons to represent the local network of ACC (Brunel, 2000). All neurons in the network receive Poisson drive at a rate of $r_{\text {ext }}=30 \mathrm{kHz}$ to reflect external inputs. All connections involving inhibitory neurons in this network were established randomly with $10 \%$ connection probability and then kept fixed. Only excitatory to excitatory (E-E) connections were subject to homeostatic structural plasticity (HSP) (Gallinaro and Rotter, 2018; Lu et al., 2019). Each excitatory neuron monitors its own firing rate using its intracellular calcium concentration and grows or retracts its spines and boutons to form or break synapses (Figure 1A, see Supplementary Materials for details). Initially, the network has no E-E connections at all, but spontaneous growth goes on until an equilibrium between network activity and structure is reached. In this equilibrium state, the E-E connectivity is at 9\%, and all excitatory neurons fire around the rate of $8 \mathrm{~Hz}$ imposed by the controller. Spiking activity is generally asynchronous and irregular. Detailed parameters of the neuron model, synapse model, and network model can be found in Extended Data Tables 1, Table 2, and Table 3. The methods employed to measure neuronal activity and connectivity in numerical experiments are described again in more detail in the Supplementary Materials. 


\section{Modeling optogenetics}

Optogenetics uses microbial opsin genes to achieve optical control of action potentials in specific neuron populations (Yizhar et al., 2011). In our current study, we used humanized channelrhodopsin-2 (hChR2), a fast light-gated cation-selective channel, to depolarize mouse pyramidal neurons (Nagel et al., 2003). The kinetics of ChR2 activation is a complicated light-dark adaptation process: Light activates and desensitizes the channels, while they recover in the dark phase (Bruun et al., 2015; Zamani et al., 2017). These state transitions have been studied in detail in computational models of ChR2 (Nikolic et al., 2013; Williams et al., 2013). It did not seem necessary, however, to include the detailed kinetics of ChR2 in our large spiking neural network with homeostatic structural plasticity. To reduce the complexity of the model and save computational power, we conceived optogenetic stimulation as an extra Poisson input of rate $r_{\text {opto }}=1.5 \mathrm{kHz}$ and weight $J_{\text {opto }}=0.1 \mathrm{mV}$. Neurons responded with an increased firing rate to this stimulation, as observed in optogenetic stimulation experiments.

\section{Numerical experimental design}

In mouse experiments, the ACC of animals were optogenetically stimulated for four consecutive days at the same time, with a duration of 30 min per day. In our computational model, we started the optogenetic stimulation after the network had reached its structural equilibrium. To avoid excessively long simulation time, we accelerated the remodeling process by employing relatively fast spine and bouton growth rates, see Figure 2 of Gallinaro and Rotter (2018) for details. The relative duration of stimulation vs. relaxation was left unchanged, however. Since the optogenetic stimulation in experiments activates a large fraction of all pyramidal neurons in ACC, we chose to stimulate half of the excitatory neurons $\left(f_{\text {opto }}=50 \%\right)$ in the model. All the model parameters are summarized in Extended Data Table 4. 


\section{Animals}

3-5 months old genetically modified mice expressing ChR2 and yellow fluorescent protein (Thy1-ChR2-YFP) in a subset of pyramidal neurons (MGI Cat\# 3719993, RRID:MGI:3719993) as well as C57BL/6J male adult mice (IMSR Cat\#JAX:000664, RRID:IMSR_JAX:000664; Charles River, L'Arbresle, France) were used in the current study. All mice were kept in a reversed day-night cycle, with food and water provided ad-libitum. Mice were firstly group caged and then single housed after the optic fiber implantation. The Chronobiotron animal facilities are registered for animal experimentation (Agreement A67-2018-38), and protocols were approved by the local ethical committee of the University of Strasbourg (CREMEAS, $n^{\circ}$ 02015021314412082).

\section{Animal experimental design}

The animal experiments' objective was to clarify the dynamic plastic phenomenon triggered by external stimulation as predicted by our computational model. We adopted an established optogenetics mouse model from our laboratory, in which the pyramidal neurons in ACC (24a/24b) were activated for four consecutive days (details see Optogenetic stimulation section below). We estimated the temporal dynamics with discrete time points, by harvesting the mouse brain tissue at $1.5 \mathrm{~h}, 24 \mathrm{~h}$, or $48 \mathrm{~h}$ after the last stimulation. As shown in our previous studies (Barthas et al., 2015; Barthas et al., 2017), sustained stimulation of the ACC induces depressive-like behavior in naïve mice. So in the current study, we used splash test and novelty-suppressed feeding (NSF) test to verify the behavioral effects of the optogenetic stimulation. In the $24 \mathrm{~h}$-post groups, we conducted splash test on the fifth day, while in the 48 h-post groups, we performed both NSF test and splash test on the fifth and sixth day and sacrificed the mice afterwards. We later estimated the temporal evolution of neural activity and connectivity indirectly by quantifying the expression of c-Fos and the spine morphology of ACC pyramidal neurons harvested at $24 \mathrm{~h}$ and $48 \mathrm{~h}$ post-stimulation. The underlying mechanism was estimated by the expression of synaptic proteins and glial markers at $1.5 \mathrm{~h}, 24 \mathrm{~h}$, and $48 \mathrm{~h}$. Before we performed all the experiments 
in Thy1-ChR2-YFP mice, we compared the efficacy of the transgenic approach with viral transfection. For the latter, we injected bilaterally AAV-CaMKII-ChR2 (H134R)-EYFP (Addgene plasmid \#26969; http://n2t.net/addgene:26969; RRID: Addgene_26969) into the ACC (details see Virus injection section in the Supplementary Materials) of C57BL/6J mice. As we observed no differences in the c-Fos activity and behavioral outcomes in two approaches after optogenetic activation, we decided to perform all the experiments in transgenic mice to reduce the number of surgery that animals go through. All mice group information was summarized in Extended Data Table 5.

\section{Stereotactic surgery}

Stereotactic surgery was conducted to inject virus and implant optic fiber into ACC. During the surgery, mice were deeply anesthetized with a mixture of zoletil $(25 \mathrm{mg} / \mathrm{kg}$ tiletamine and $25 \mathrm{mg} / \mathrm{kg}$ zolazepam) and xylazine $(10 \mathrm{mg} / \mathrm{kg}$ ) (Centravet, Taden, France; i.p. injection) and locally anesthetized by bupivacaine (Mylan, The Netherlands; $0.5 \mathrm{mg} / \mathrm{mL}$; subcutaneous injection, $1 \mathrm{mg} / \mathrm{kg}$ ). The coordinates of the injection/implantation site are $+0.7 \mathrm{~mm}$ from bregma, lateral: $\pm 0.3 \mathrm{~mm}$, dorsoventral: $-1.5 \mathrm{~mm}$ from the skull (Barthas et al., 2015; Sellmeijer et al., 2018).

\section{Optic fiber implantation}

We inserted $1.7 \mathrm{~mm}$-long LED optic fiber (MFC_220/250-0.66_1.7mm_RM3_FLT, Doric Lenses, Canada) unilaterally (left or right) in C57BL/6J mice two weeks after the virus injection or directly in naïve Thy1-ChR2-YFP mice. The fiber was inserted into ACC for $1.5 \mathrm{~mm}$ deep with reference to the skull. The metal end was fixed onto the skull by superglue and dental cement, and then the skin was stitched. For stimulation, we used blue light (460 nm wavelength) and the light intensity of optic fibers used in the current study ranged from $1.7 \mathrm{mV}$ to $6 \mathrm{mV}$. 


\section{Optogenetic stimulation}

After the optic fiber implantation, we individually housed the mice to avoid possible damage to the implant. After seven days of recovery, we started the optogenetic stimulation protocol on freely moving mice in their home cages. Optogenetic stimulation took place on four consecutive days for $30 \mathrm{~min}$. Stimulated mice received repetitive stimuli sequences of ten seconds consisting of eight seconds at $20 \mathrm{~Hz}$ with $40 \mathrm{~ms}$ pulses and two seconds without stimulation. We did not observe the effects of light on the behaviors in gene-matched wild type mice (Extended Data Figure 2-2). So we used transgenic mice for all experiments and kept the light off for the sham groups. At the end of stimulation, all mice were handled again and unplugged from the cable.

\section{Behavioral tests}

We performed all the behavioral tests during the dark phase under red light. Splash test (Nollet et al., 2013) and novelty-suppressed feeding (NSF) test (Samuels and Hen, 2011) were used to evaluate depressive-like behaviors. In the splash test, we sprayed $15 \%$ sucrose solution onto the coat of the mice and recorded the total grooming time for each mouse during the following $5 \mathrm{~min}$. The NSF test was conducted on a different day of splash test and we removed the food pellets $24 \mathrm{~h}$ before testing. During the test, we put each mouse into an open field, where a food pellet was placed in the middle, and recorded the time delay necessary for each mouse to touch and eat the pellet (within $5 \mathrm{~min}$ ).

\section{Verification of injection site and tissue harvesting}

Mice were perfused with cold $4 \%$ paraformaldehyde $(\mathrm{PFA})$ in $1 \times$ phosphate buffer $(\mathrm{PB}$, 0.1 M, pH 7.4), under Euthasol Vet (intraperitoneal injection, $2 \mu \mathrm{L} / \mathrm{kg}$; TVM, UK) overdose anesthesia. The details of timing and pump speed can be found in the Supplementary Materials. Frontal sectioning of the brains ( $40 \mu$ m-thick for immunohistochemical staining and $300 \mu \mathrm{m}$-thick for microinjection) was performed on a vibratome (Leica-VT1000s, Rueil-Malmaison, France). The injection or implantation site of each perfused mouse was 
checked under the microscope.

\section{Immunohistochemical staining}

We did fluorescent staining to examine the expression of c-Fos, VGLUT1, PSD-95, Neurogranin, and GFAP (see Extended Data Table 6 for the antibody concentrations). We used sections ranging from $1.42 \mathrm{~mm}$ to $-0.23 \mathrm{~mm}$ away from Bregma, with a distance $160 \mu \mathrm{m}$ in between. The sections were firstly washed in $1 \times \mathrm{PBS}(3 \times 10 \mathrm{~min})$ and then blocked at room temperature (RT) with $5 \%$ donkey serum in $0.3 \%$ PBS-T (1 h). Later the sections were incubated at $4{ }^{\circ} \mathrm{C}$ with corresponding primary antibody and $1 \%$ donkey serum in $0.3 \%$ PBS-T overnight. Sections were rinsed with $1 \times$ PBS $(3 \times 10 \mathrm{~min})$ in the next morning, incubated with secondary antibody in $0.3 \%$ PBS-T at RT $(2 \mathrm{~h})$, and rinsed again with $1 \times$ PBS $(3 \times 10 \mathrm{~min})$. Sections were mounted on gelatin-coated slides, air-dried, and coverslipped with Vectashield H-1000 (Vector Laboratories, Germany).

We stained IBA1 with 3,3'-Diaminobenzidine (DAB, Sigma, US). Sections were selected, washed, blocked, and treated with primary and secondary antibodies as described above. Then the sections were rinsed with $1 \times$ PBS $(3 \times 10 \mathrm{~min})$ and incubated with avidin-biotin-peroxidase complex (ABC Elite, Vector Laboratories, Germany; 0.2\% A and $0.2 \% \mathrm{~B}$ in $1 \times \mathrm{PBS})$ at RT $(1.5 \mathrm{~h})$. Later the sections were rinsed with $0.05 \mathrm{M}$ Tris$\mathrm{HCl}$ buffer $(\mathrm{TB} ; \mathrm{pH} 7.5 ; 3 \times 10 \mathrm{~min})$. Peroxidase revelation was achieved by incubation shortly $(20 \mathrm{~s})$ with a mixture of $0.025 \%$ DAB and $0.0006 \% \mathrm{H}_{2} \mathrm{O}_{2}$ in $0.05 \mathrm{M}$ TB. Sections were carefully rinsed with TB $(2 \times 10 \mathrm{~min})$ and $1 \times$ PBS $(2 \times 10 \mathrm{~min})$ to cease the reaction. All sections were mounted and air-dried, then dehydrated in graded alcohol baths $(1 \times 5 \mathrm{~min}$ in $70 \%, 1 \times 5 \mathrm{~min}$ in $90 \%$, and $2 \times 5 \mathrm{~min}$ in $100 \%$ ), cleared in Roti-Histol (Carl Roth, Germany), and coverslipped with Eukitt.

\section{Microinjection}

We used microinjection and confocal microscope (Dumitriu et al., 2011) to visualize and quantify the neural morphology at $24 \mathrm{~h}$ and $48 \mathrm{~h}$ post-stimulation. The sections were se- 
lected within the distance of approximately $\pm 0.4 \mathrm{~mm}$ anterior-posterior (AP) away from the optic fiber. The injection was done only into the pyramidal neurons from layer $2-3$ of ACC (24a/24b) from both hemispheres. The injection pipettes were pulled from glass capillaries with filament, with a final resistance around $150 \mathrm{M} \Omega$. We filled the pipette with red fluorescent dye solution Alexa 568 hydrazide (\#A10441, Thermo Fisher, USA) in filtered $1 \times$ PBS $(1: 40)$. We performed microinjection under the microscope of a patchclamp set-up. During injection, we penetrated the pipette tip into the soma and switched on the current to $-20 \mathrm{pA}$ to drive the dye diffusion for $20 \mathrm{~min}$. Later we switched off the current but left the pipette tip inside the soma for another $5 \mathrm{~min}$ to fill the dendrite and spines. All the sections were retrieved and covered with Vectashild H-1000 (Vector Laboratories, Germany) for confocal microscope imaging. We checked all the injected neurons for YFP signal; only neurons with YFP signal were identified as pyramidal neurons and selected for further analysis.

\section{Microscope imaging}

To analyze the morphological features, we took z-stacked images of microinjected neurons (with step size $0.2 \mu \mathrm{m}-0.3 \mu \mathrm{m}$ sampled by the software Leica SP8 LAXS 3.5.6) with confocal microscope Leica SP8 (Leica Microsystems, Germany). Whole neuron structure were imaged with objective HC PL APO CS2 $63 \times / 1.40$, with pixel resolution $0.2 \mu \mathrm{m}$ per pixel. If not stated otherwise, for excitation we used a pulsed laser (White Light; $488 \mathrm{~nm}$ ). The dendrite segments, from apical and basal dendrites of each neuron, were imaged with objective HC PL APO CS2 $63 \times / 1.40$ as well, but with pixel resolution $0.05 \mu \mathrm{m}$ per pixel. Secondary to third level dendrite segments with less overlap and clear background were selected.

To quantify the expression of c-Fos, VGLUT1, PSD-95, neurogranin, and GFAP in the ACC, we imaged epifluorescent signals of stained sections with Morpho Strider on Zeiss Imager2 (Carl Zeiss, Germany) with $2.5 \times$ objective and pixel resolution $0.35 \mu \mathrm{m}$ per pixel. To achieve better resolution of representative images, we also imaged the sections at the 
middle focal plane with a confocal microscope Leica SP8 (Leica Microsystems, Germany; software Leica SP8 LAXS 3.5.6) with objectives HCX PL Fluotar $5 \times / 0.15$ and HC PL APO CS2 $40 \times / 1.30$, with pixel resolution $0.5 \mu \mathrm{m}$ and $0.1 \mu \mathrm{m}$ per pixel, respectively. The bright-field images of DAB-stained IBA1 were acquired with a NikonEclipse E600 microscope with $4 \times$ and $40 \times$ objectives (MBF Bioscience, USA; software Neurolucida 2019), with pixel resolution $0.1 \mu \mathrm{m}$ and $2.0 \mu \mathrm{m}$ per pixel.

\section{D reconstruction and analysis of dendritic morphology}

Firstly, after imaging, we deconvolved our confocal z-stack images with Huygens Professional 19.04 (Scientific Volume Imaging, The Netherlands) to restore the object from the acquired image through knowledge of the point spread function (PSF) and noise. $3 \mathrm{D}$ reconstruction and morphological analysis were later performed on the deconvoluted images.

For each pyramidal neuron, we reconstructed the soma and its dendritic tree with Imaris 9.5.1 (ImarisXT, Bitplane AG, Switzerland). Based on the reconstructed data, the dendritic tree structure was represented by Sholl intersections (Sholl, 1953) at different radiuses. The order of each dendrite segment and its corresponding length and average diameter were also estimated. We further used Fiji (ImageJ, Fiji) to measure the soma size of each neuron on its z-projected image.

We reconstructed the dendritic shafts and spines with Imaris 9.5.1 again for selected dendrite segments at high resolution. We also classified the spine classes (filopodia, longthin, stubby, and mushroom) based on their morphological features with the Imaris Spines Classifier package. The criteria of spine classification were summarized in Extended Data Table 7. We harvested the overall spine density of each segment and the spine density of each spine class based on the reconstructed data. The spine head volume of individual spines was also estimated. 


\section{Quantifying immunohistochemical staining images}

Visually inspection showed the expression of marker proteins was not homogeneously distributed in ACC but constrained to the vicinity around the optic fiber. To reflect such a pattern, we systematically analyzed the expression of c-Fos, VGLUT1, PSD-95, Neurogranin, GFAP, and IBA1 in both hemispheres at different distances to the optic fiber at $1.5 \mathrm{~h}, 24 \mathrm{~h}$, and $48 \mathrm{~h}$ post-stimulation.

We firstly arranged the corresponding epifluorescent images (obtained under $2.5 \times$ objective) or bright-field images (obtained under $4 \times$ objective) of each marker for each mouse in sequential order. The Bregma level of each section was identified in reference to the Mouse Brain Atlas (Franklin et al., 2008). Later we checked the implantation site for each section. Sections with a clear trace of implantation were marked as distance zero. Sections at a more anterior position than the distance zero were labeled with a negative sign $(-)$, while posterior sections were labeled with a positive sign $(+)$. In the end all sections were classified into five distance groups and their average distances were noted as $-0.4 \mathrm{~mm},-0.2 \mathrm{~mm}, 0 \mathrm{~mm},+0.2 \mathrm{~mm}$, and $+0.4 \mathrm{~mm}$. Both hemispheres were also carefully identified as the ipsi- or contralateral side in reference to the implantation site.

To quantify the signal intensity of markers on each section, we created two same-sized masks $(700 \mu \mathrm{m} \times 700 \mu \mathrm{m})$ on both hemispheres with Fiji. For c-Fos and IBA1, we counted signal positive cell numbers within each section's masks, while for VGLUT1, PSD-95, neurogranin, and GFAP, we quantified the fluorescent intensity within the masks.

For Neurogranin, in addition to the overall signal intensity, we also quantified its relative intensity in soma and dendrites to infer its cellular translocation. Sections within $0.1 \mathrm{~mm}$ anterior-posterior to the optic fiber were selected. We first drew the shape of soma and measured its fluorescent signal intensity and area size. Then we moved the mask to the neighboring area around the soma and measured the fluorescent intensity of the samesized area as a reference. Five random selections were measured in the adjacent regions and averaged to serve as the final equivalent dendrites intensity. We normalized the signal intensity of soma by its equivalent dendrites intensity as the relative soma intensity. 


\section{Statistical analysis}

We have different types of data in the current study, non-clustered independent data and nested data. Independent measurement, such as behavioral data, was contributed only once by each mouse. We used the non-parametric Mann Whitney U test to examine if the optogenetic stimulation triggered significant behavioral alterations.

Some datasets, such as the signal intensity of immunohistochemical staining and the neural morphology, are highly nested. In the staining experiments, each mouse contributed multiple brain sections in five distance groups; for the morphological data, each mouse contributed several neurons, and each neuron further contributed multiple dendrite segments. We therefore used linear mixed effects model (LMM) in R (R Core Team, 2019) to assess the effects of experimental variables, while accounting for the nested residual structure, that is caused by our design. We used the lme4 package (Bates et al., 2015) and applied glmer function (GLMM) to model cell counting data. Our null hypothesis is that there is no significant difference between the sham and the stimulated mice; If there is a difference, the discrepancy remains at a similar level on the ipsi- and contralateral hemisphere, or stayed at the same level for five distance groups. So in the model, we set the main effects of stimulation, distance to the optic fiber, implantation site, and their interaction effects as fixed effects, and neuron ID or/and animal ID as random effects. Three discrete time points were separately analyzed. We used a three-step process to select the best-fit combination of random and fixed effects structures following Zuur et al. (2009). MuMIn (Barton, 2019) was used for model selections based on Akaike's information criterion (AIC) value. All final models were checked in terms of homogeneous and normally distributed residuals, using diagnostic plots. We further checked final models for over-dispersion. Detailed model structures and the model selection and validation processes were described in the Supplementary Materials. All the R scripts of LMM and GLMM could be found under the following link: https://github.com/ErbB4/LMM-GLMM-R-plasticity-paper

The significance of fixed effects was tested by extracting effect strengths of each pa- 
rameter, including their confidence intervals; $p<0.05, p<0.01$, and $p<0.001$ were used to indicate $95 \%$ CI, 99\% CI, or $99.9 \%$ CI of the estimated coefficient does not cross zero. If not stated otherwise, $*$ denoted the main effect of optogenetic stimulation (sham/stimulated) + denoted the main effect of stimulation side (ipsi/contra to the optic fiber), and \# denoted the main effect of distance to the optical fiber. Significant interaction effects were not denoted but stated in the main text. 'n.s.' denoted neither main effects nor interaction effects were significant. 


\section{Results}

\section{Computational model predicted homeostatic responses upon op- togenetic stimulation}

To represent ACC, the target region of the optogenetic stimulation, we established an inhibition-dominated spiking neural network subject to a linear HSP rule (Figure 1A). Based on the HSP rule, the connections among the excitatory neurons grew from zero to an equilibrium state in the presence of background inputs. The optogenetic stimulation was later introduced to half of the excitatory population and then terminated (light blue shaded areas in Figure 1B). The application and termination of the stimulation perturbed the neural activity and triggered HSP-based synapse turnover (blue curves in Figure 1B). Homeostasis of neural activity was re-achieved by the re-growth of synapses after the stimulation was terminated, but the network architecture did not return to the state before stimulation. Compared to the pre-stimulation network, although the average number of synapses per neuron was not altered, the source of synapses to the stimulated neurons was reorganized. Neurons being stimulated were connected less with the nonstimulated neurons but wired more with other stimulated neurons (Figure 1C). As a result, the connectivity among the stimulated neurons remained elevated after the firing rate homeostasis was re-achieved (Figure 1D). This HSP-mediated remodeling process has been extensively discussed in our previous work (Lu et al., 2019).

Based on this model, we applied a customized protocol to predict the HSP-mediated phenomenon involved in our in vivo experiments. The relative duration of the stimulation period and the inter-stimulation-interval was adjusted to match the applied stimulation duration $(30 \mathrm{~min} /$ day $\times 4$ days $)$, and the network was relaxed for $48 \mathrm{~h}$ after the last stimulation session. As shown in Figure 1E, the connectivity among the stimulated neurons accumulated through repetitions and gradually evoked stronger neural activity in response to the same stimulation. The small insets in Figure 1E showed the predicted dynamics within $48 \mathrm{~h}$ after the last stimulation session (S4). Optogenetic stimulation induced hy- 
peractivity in the stimulated neurons, but once the stimulation was terminated, the neural activity underwent transient reduction and quickly returned to baseline. In parallel, the connectivity among the stimulated neurons underwent homeostatic increase and decayed slowly to baseline. We thus hypothesized that if homeostatic structural plasticity dominated the plastic regulation after the optogenetic stimulation, we should observe the neural activity of the stimulated neurons gradually restore to baseline after the stimulation, while the spine density and the expression of synaptic proteins of the stimulated neurons, as indirect measurements of connectivity, should undergo rise and decay.

\section{Optogenetic activation of ACC (24a/24b) pyramidal neurons trig-}

\section{gered cortical hyperactivity and behavioral alterations}

To clarify the dynamics of homeostatic plasticity predicted by our computational model in vivo, we adopted the optogenetic mouse model previously published in our laboratory (Barthas et al., 2015; Barthas et al., 2017) to repetitively activate ACC pyramidal neurons. We firstly compared the viral transfection and transgenic approaches (Extended Data Figure 2-1A-E). We previously showed with the transgenic approach that there was an increased c-Fos expression at $1.5 \mathrm{~h}$ after the stimulation (Barthas et al., 2015) and here we also reproduced the results with viral injection approach (Extended Data Figure 21F-G; Figure 2G, left panel). Besides the cortical hyperactivity, both approaches induced a depressive-like phenotype in mice at $24 \mathrm{~h}$ and $48 \mathrm{~h}$ post-stimulation (Extended Data Figure 2-1H-I; Figure 2E-F). To avoid double surgeries, we decided to continue with transgenic mice throughout the current study. We also confirmed that light did not trigger behavioral alterations (Extended Data Figure 2-2).

In two other batches of transgenic mice, we also examined the c-Fos expression at $24 \mathrm{~h}$ and $48 \mathrm{~h}$ post-stimulation and observed no difference between the stimulated and sham mice (Figure 2G, middle and right panels). These data suggested optogenetic stimulation triggered hyperactivity in ACC and the neural activity was restored to baseline level at $24 \mathrm{~h}$ and $48 \mathrm{~h}$ post-stimulation. 
To capture the temporal evolution of homeostatic structural plasticity, we stimulated transgenic Thy1-ChR2-YFP male adult mice and harvested their brains at $1.5 \mathrm{~h}$, $24 \mathrm{~h}$, and $48 \mathrm{~h}$ post-stimulation for further experiments (Figure 2A-C). Mice used for immunohistochemical staining experiments showed depressive-like behaviors at $24 \mathrm{~h}$ and $48 \mathrm{~h}$ post-stimulation, as shown by decreased grooming behaviour in splash test and increased latency to bite in the NSF test (Figure 2D-F). All mice group information and experimental design were summarized in Extended Data Table 5.

\section{Dendritic tree structure was not drastically affected by the op- togenetic stimulation at $24 \mathrm{~h}$ and $48 \mathrm{~h}$}

Based on the homeostatic assumptions, we first focused on the possible alteration of the neuronal morphology at $24 \mathrm{~h}$ and $48 \mathrm{~h}$ when the neural activity already returned to baseline. We stimulated mice the same way as described above and harvested their brains at $24 \mathrm{~h}$ or $48 \mathrm{~h}$ post-stimulation. As shown in Figure 3A, mice showed depressivelike behavior as expected ( $p=0.004$ for 24 h-post group, $p=0.012$ and $p=0.006$ for 48 h-post group, Mann Whitney U test). We injected red fluorescent dye into pyramidal neurons selected from the area around the optic fiber to visualize and analyze the neuronal morphology.

Neural dendritic structure at $24 \mathrm{~h}$ and $48 \mathrm{~h}$ was visualized as in Figure 3B. Pyramidal neurons from both ipsi- and contralateral ACC were collected (Figure 3C). The soma size and dendritic tree structure evaluated by Sholl intersections were not changed by the optogenetic stimulation (Figure 3D-E). No remarkable changes were detected in neither dendritic length nor average dendritic diameter, except that some dendritic segments showed a reduction or increase in dendritic diameter (Extended Data Figure 3-1). Our data suggested, except for local dendrite diameter changes, no drastic dendritic tree structure and soma size inflation or shrinkage of pyramidal neurons in the vicinity of the optic fiber were induced by the optogenetic stimulation. 


\section{Optogenetic stimulation induced the opposite spine morphologi- cal changes at $24 \mathrm{~h}$ and $48 \mathrm{~h}$}

To further analyze dendritic spine density, we sampled several secondary to third level apical and basal dendritic segments from each neuron and did the 3D reconstruction of spines (Figure 4A-B). Besides spine density, we also evaluated the spine head volume and classified different types of spines such as filopodia, long-thin, stubby, and mushroom.

As shown in Figure 4C-D, the overall spine density was increased at $24 \mathrm{~h}(p<0.05$, LMM) and decreased at $48 \mathrm{~h}(p<0.05, \mathrm{LMM})$ post-stimulation. The apical spine density showed the same tendency but the changes were not statistically significant; the basal dendrites showed significant spine density alterations ( $p<0.05$ and $p<0.05$, LMM). Analysis by spine type suggested subtle changes in different spine types (Figure 4E-F). At $24 \mathrm{~h}$, the spine density of filopodia and stubby type was increased in both apical $(p<0.05$ and $p<0.05$ respectively, LMM) and basal dendrites ( $p<0.05$ and $p<0.05$, LMM). At $48 \mathrm{~h}$, the spine density of long-thin type was reduced in apical dendrites $(p<0.05$, LMM), while the stubby and mushroom type were reduced in basal dendrites $(p<0.05$ and $p<0.05$ respectively, LMM). These data suggested optogenetic stimulation triggered spinogenesis and spine retraction in both apical and basal dendrites at $24 \mathrm{~h}$ and $48 \mathrm{~h}$ post-stimulation respectively.

In addition, spine head volume data (Figure 4G-H) showed different regulation in apical and basal dendrites. At $24 \mathrm{~h}$, the head volume distribution of long-thin spines in the apical dendrites was right-shifted to larger mean values by the optogenetic stimulation $(p<0.05, \mathrm{LMM})$ while no changes were detected in the head volume of basal dendrite spines. At $48 \mathrm{~h}$, the spine head volume of mushroom spines in basal dendrites was leftshifted to smaller mean values by the optogenetic stimulation $(p<0.05, \mathrm{LMM})$, whereas the apical dendrites showed no significant difference. Our data suggested in addition to spine density changes, optogenetic stimulation induced spine enlargement and shrinkage at $24 \mathrm{~h}$ and $48 \mathrm{~h}$ post-stimulation respectively. 


\section{VGLUT1 and PSD-95 in ACC showed distance- and time- de- pendent regulation by optogenetic stimulation}

As the optogenetic stimulation altered the spine morphology of the pyramidal neurons, we wondered what the corresponding molecular signatures of the structural alterations are. The expression of both presynaptic VGLUT1 and postsynaptic PSD-95 was evaluated at $1.5 \mathrm{~h}, 24 \mathrm{~h}$, and $48 \mathrm{~h}$ (Extended Data Table 5). We conducted immunohistochemical staining to detect the expression of synaptic proteins in the ipsi- and contralateral hemispheres of ACC sections with reference to the hemisphere where the optic fiber was implanted. Frontal-sectioned brain slices in both sham and the stimulated mice were organized based on their distance away from the optic fiber.

The representative fluorescent staining of VGLUT1 was organized by distance and by time in Extended Data Figure 5-1. Intensity quantification summarized in Figure 5A-B showed that optogenetic stimulation did not trigger significant alteration at $1.5 \mathrm{~h}$, while significant upregulation was observed in the stimulated mice compared to sham mice at $24 \mathrm{~h}(p<0.001, \mathrm{LMM})$. Further examination of interaction effects confirmed stronger upregulation in the ipsilateral side $(p<0001, \mathrm{LMM})$ and in the sections close to the optic fiber $(p<0.001, \mathrm{LMM})$ in the stimulated mice. At $48 \mathrm{~h}$, no more significant difference was detected between the stimulated and sham mice. Our data at discrete time points suggested that optogenetic stimulation altered VGLUT1 expression in a time-dependent manner. Indeed, the upregulation was observed after $1.5 \mathrm{~h}$, peaked around $24 \mathrm{~h}$, and returned to baseline at $48 \mathrm{~h}$ post-stimulation, while the stimulation effects were constrained to areas around the optic fiber.

Similar expression pattern was observed with PSD-95 staining (Extended Data Figure 5-2, Figure 5C-D). At $1.5 \mathrm{~h}$, no significant changes were induced by the stimulation. At $24 \mathrm{~h}$, enhanced expression of PSD-95 in ACC was observed in the stimulated mice $(p<0.01, \mathrm{LMM})$ and specifically in the ipsilateral hemisphere $(p<0.001, \mathrm{LMM})$ and sections close to the optic fiber $(p<0.001, \mathrm{LMM})$. At $48 \mathrm{~h}$, although the effect size was small, the PSD-95 expression of the stimulated mice declined to a lower level than 
sham $(p<0.01$, LMM). Our data suggested a similar time-dependent manner of PSD95 upregulation as VGLUT1 after the optogenetic stimulation: upregulation at $24 \mathrm{~h}$ and downregulation at $48 \mathrm{~h}$.

\section{Neurogranin showed time-dependent translocation by the opto- genetic stimulation}

Since PSD-95 is expressed in the postsynaptic membrane of glutamatergic synapses in both excitatory and inhibitory neurons (Zhang et al., 1999), we studied another postsynaptic protein, neurogranin, which is exclusively expressed in the pyramidal neurons (Singec et al., 2004). Despite the fact that the same type of quantification and analysis procedures were applied to neurogranin stained ACC sections, no time-dependent or distance-dependent alterations of neurogranin was observed (Extended Data Figure 6-1, Figure 6A-B).

Considering that neurogranin is expressed in both soma and spines, and neuronal stimulation could drive the translocation of neurogranin from soma to dendrites (Huang et al., 2011), we suspected that the optogenetic stimulation might fail to trigger neurogranin upregulation but induced the cellular translocation. Consequently, we selectively analyzed the high magnification images of sections within $0.1 \mathrm{~mm}$ anterior-posterior to the optic fiber. We quantified the relative fluorescent intensity of neurogranin in the soma against the region round soma, which was considered as the equivalent area of interconnected dendritic trees and extracellular matrix (Figure 6C-D). Pyramidal neurons from the three sham groups all showed a high soma concentration. After the stimulation, the relative signal intensity of soma was slightly increased at $1.5 \mathrm{~h}(p<0.001$, Mann Whitney U test), decreased to a level lower than 1 at $24 \mathrm{~h}(p<0.001$, Mann Whitney U test), and recovered to a level above 1 but lower than the sham group at $48 \mathrm{~h}(p<0.001$, Mann Whitney U test). These data supported our hypothesis that the optogenetic stimulation did not trigger neurogranin upregulation, but induced translocation with time: concentrated in soma at $1.5 \mathrm{~h}$, translocated to dendrites at $24 \mathrm{~h}$, and recovered at $48 \mathrm{~h}$. The modulation 
of neurogranin and PSD-95 orchestrated with VGLUT1, suggested synaptic transmission was enhanced at $24 \mathrm{~h}$ and decayed afterwards.

\section{Glial responses were involved in homeostatic plasticity induced by the optogenetic stimulation}

Glial cells were reported to participate in neural plasticity and maintain extracellular homeostasis (Dissing-Olesen et al., 2014; Haydon and Nedergaard, 2015). So we expected simultaneous glial responses that emerged with the alterations of spine morphology and synaptic proteins. Glial fibrillary acidic protein (GFAP) was stained as the markers for activated astrocytes (Hol and Pekny, 2015), and ionized calcium-binding adaptor molecule 1 (IBA1) was stained for both inactive and active microglia (Ohsawa et al., 2004).

The fluorescent staining of GFAP was organized by distance and by time in Extended Data Figure 7-1. Our statistics analysis showed optic fiber implantation triggered astrocytes reactivation in both sham and stimulated mice, but the stimulation further enhanced the reactivation in a distance-dependent manner throughout $48 \mathrm{~h}$ post-stimulation (Figure 7A-B). Similar results were observed in IBA1 signal (Extended Data Figure 7-2), that optogenetic stimulation induced significant enhancement of IBA1 expression at $24 \mathrm{~h}$ and $48 \mathrm{~h}$ post-stimulation $(p<0.001$ and $p<0.001$, GLMM) in the ipsilateral hemisphere and in the sections close to the optic fiber (Figure 7C-D). Since IBA1 labels microglia regardless of its activation state, the upregulation of IBA1 suggested microglia proliferation. Our data suggested that optogenetic stimulation triggered astrocytes reactivation and microglia proliferation throughout $48 \mathrm{~h}$ post-stimulation. 


\section{Discussion}

Inspired by the modeling study (Figure 1), we have systematically investigated the plastic responses of neocortical networks upon optogenetic stimulation in an in vivo mouse model. We found that the activation of a subset of excitatory neurons in ACC over four consecutive days triggered substantial plastic alterations. The temporal profile of specific morphological and molecular changes over $48 \mathrm{~h}$ post-stimulation was roughly in line with predictions from a homeostatic plasticity model: While the hyperactivity of stimulated neurons is quickly restored to baseline after stimulation, the connectivity among stimulated neurons first overshoots and then slowly decreases back to baseline (Figure 8, red and blue curves). This temporal profile is a characteristic fingerprint of homeostatic control (Gallinaro and Rotter, 2018; Lu et al., 2019; Gallinaro et al., 2020). We quantified an indicator of neuronal activity (c-Fos) 1.5, 24, and 48 hours after the last stimulation. We observed increased activity levels at $1.5 \mathrm{~h}$, but only baseline activity at $24 \mathrm{~h}$ and $48 \mathrm{~h}$ post-stimulation. To account for plastic changes, we assessed the morphology of dendritic spines on pyramidal neurons, the expression levels of synaptic proteins (VGLUT1, PSD-95, neurogranin) and glial markers (GFAP, IBA1). We found that spine density and spine head volume of stimulated neurons were increased $24 \mathrm{~h}$ and decreased $48 \mathrm{~h}$ after stimulation, as compared to sham. The same temporal profile of upregulation and downregulation was observed for synaptic proteins VGLUT1 and PSD-95. The postsynaptic protein neurogranin was translocated from the soma to the dendrites of stimulated neurons at $24 \mathrm{~h}$, and it was restored at $48 \mathrm{~h}$. Indicators of astrocyte activation (GFAP) and microglia proliferation (IBA1) were elevated throughout the observed post-stimulation period.

In previous modeling work, Lu et al. (2019) have investigated homeostatic network remodeling after transcranial direct current stimulation (tDCS). Although our current experimental work employs optogenetic stimulation instead, taken with a grain of salt, both methods perturb the activity of the network in similar ways. According to our model, this induces a plastic change of network structure that counteracts the perturbation to 
restore the equilibrium. Different from the tDCS study, we assumed here that a rather large fraction of ACC excitatory neurons is activated by light. As usual, the stimulated neurons first randomly break synapses during the stimulation and then form a surplus of intra-group synapses with a delay. However, in this configuration, numerous inter-group synapses are also broken, and the non-stimulated neurons are deprived of their excitatory inputs. Therefore, they rewire and seek inputs from other non-stimulated neurons. As a result, we even obtain enhanced connectivity among the non-stimulated neurons. This indirect effect is not prominent, if the stimulated group is small, as it was the case in our tDCS model. The exact time course of the transient growth and neural activity restoration follows from the negative feedback dynamics imposed by the homeostatic controller.

Alterations in spine density and connectivity as a result of external stimulation have previously been reported for contextual fear conditioning (CFC). Ryan et al. (2015) labeled the memory engram corresponding to the context and found that $24 \mathrm{~h}$ post-CFC both spine density and connectivity of engram cells increased as compared to sham. We observed similar results in experiments and our homeostatic control model can also explain the observed structural dynamics. As indicated by c-Fos expression, the stimulus-induced hyperactivity of neurons was restored to baseline $24 \mathrm{~h}$ post-stimulation. The density of spines on stimulated neurons, however, was still elevated after $24 \mathrm{~h}$ and decreased to a lower level after $48 \mathrm{~h}$. Although we did not measure the connectivity among stimulated neurons directly, our data qualitatively matched the time course of stimulationtriggered connectivity transients seen in computer simulations. It is a specific feature of our model that the overshoot and decay of spine density happens after neural activity is back to baseline. Besides, we found in our experiments that spine head volume underwent similar transients. As spine head volume is correlated with the amount of PSD-95 and the strength of a synapse (Matsuzaki et al., 2004; Broadhead et al., 2016; Lisman, 2017), the homeostatic regulation of synaptic strength represents a plausible interpretation of our findings.

A synapse in our structural plasticity model consists of a presynaptic bouton and a postsynaptic spine, whose growth and retraction are independently controlled in our 
model (Gallinaro et al., 2020). As a proxy for the number of mature synapses in mouse experiments, we quantified the expression of VGLUT1 and PSD-95. VGLUT1 is the glutamate transporter protein which governs quantal glutamate content of individual synaptic vesicles (Fremeau et al., 2004; Wilson et al., 2005). Therefore, any change in VGLUT1 indicates a change of glutamate release in synapses, corresponding to the presynaptic strength accumulated over many neurons. PSD-95 is a scaffold protein in the postsynaptic density which organizes the distribution of AMPA receptors (Chen et al., 2011; Zhang et al., 1999). As it is correlated with spine head volume and functional strength of synapses (Cane et al., 2014; Matsuzaki et al., 2004), we used it to indirectly assess the functional aspects of spines. We observed that the expression of PSD-95 was upregulated at $24 \mathrm{~h}$ and decayed to a level slightly below baseline at $48 \mathrm{~h}$ post-stimulation. The temporal profile of changes was very similar for density and volume of dendritic spines, which points to a coordinated mechanism to regulate postsynaptic transmission. VGLUT1 showed a temporal profile similar to PSD-95 in experiments. Although a coordinated turnover of boutons and spines was reported previously (Becker et al., 2008), the exact rules of their regulation are unknown. In our model, we assumed the same growth rule for both boutons and spines. This is in line with experimental observations suggesting that pre- and postsynaptic plasticity are regulated in an orchestrated way (Ehrlich et al., 2007; Letellier et al., 2019; Sanderson et al., 2020). The upregulation of VGLUT1 and PSD-95 at $24 \mathrm{~h}$ indicates that the functional synaptic transmission among the stimulated neurons is also enhanced.

In contrast to PSD-95, neurogranin is a calmodulin-binding protein exclusively expressed in the soma and dendrites of excitatory neurons (Singec et al., 2004). We found that somatic neurogranin was slightly increased at $1.5 \mathrm{~h}$, strongly reduced at $24 \mathrm{~h}$, and almost recovered to normal levels at $48 \mathrm{~h}$ post-stimulation. Previous in vitro studies reported a translocation of neurogranin from the cell plasma to the nucleus (Garrido-García et al., 2009) or from the soma to dendrites (Huang et al., 2011) within pyramidal neurons upon synaptic stimulation. According to Huang et al. (2011), the translocation of neurogranin is a necessary prerequisite for the translocation of calmodulin, a protein which 
constantly senses the intracellular calcium concentration and conveys this information as a conformational change (Xia and Storm, 2005). Functionally speaking, neurogranin lowers the threshold for the induction of LTP (Zhong and Gerges, 2012). Our results hint at the induction of LTP in spines mediated by calcium and triggered by optogenetic stimulation. This aligns well with the idea of calcium-based homeostatic control, and with the observed upregulation of PSD-95 and spine head volume at $24 \mathrm{~h}$ post-stimulation.

Astrocytes and microglia also actively participate in the homeostatic regulation of network remodeling. We observed increased numbers of IBA1-positive cells and enhanced GFAP expression throughout $48 \mathrm{~h}$ after stimulation. This suggests that both microglia and astrocytes were activated at least between $90 \mathrm{~min}$ and $48 \mathrm{~h}$ post-stimulation. Microglia and astrocyte activation was classified as either "harmful" (M1 and A1) or "beneficial" (M2 and A2). Harmful glia activation degenerates neurons, whereas beneficial activation helps to clean up extracellular chemicals and fosters spinogenesis (Zamanian et al., 2012; Liddelow and Barres, 2017). According to our data, the structure of dendritic trees, size of cell somas, and c-Fos expression were all not very strongly affected by optogenetic stimulation. Therefore, it seems unlikely that glia activation caused excitotoxicity and apoptosis (Zhang et al., 2002). It is possible that microglia and astrocyte activation triggered by optogenetic stimulation helps to maintain chemical homeostasis (Jo et al., 2014) and fosters morphological changes of spines (Weinhard et al., 2018).

Our study specifically casts light on the relation between ACC hyperactivity, synaptic plasticity, and depressive-like behavior. ACC is a hub for negative affects, pain, and their comorbidity (Humo et al., 2019). Chronic pain can induce hyperactivity and synaptic potentiation in ACC, along with anxiodepressive behavior in mice (Sellmeijer et al., 2018; Koga et al., 2015). ACC hyperactivity artificially induced by optogenetic stimulation also generates depressive-like behavior (Barthas et al., 2015; Barthas et al., 2017). It is unclear, however, whether changes in neuronal activity, spine morphology, and depressivelike behavior develop in parallel due to a common condition, or whether there are causal links between individual factors (Gipson and Olive, 2017). In our experiments, neural activity quickly decayed to baseline after stimulation, but the mice exhibited sustained 
depressive-like behavior; Synaptic plasticity (spine morphology and synaptic proteins) also showed transient changes, suggesting a mutual interdependency of these phenomena. Generally, the emergence of depressive-like behavior induced by optogenetic stimulation always lagged behind ACC hyperactivity. It only emerged after several stimulation sessions and persisted for around two weeks after the stimulation was terminated (Barthas et al., 2017). This evidence suggests that depressive-like behavior is mediated by some persistent changes that depend on the accumulated effects of neural activity. Network remodeling would be a candidate process, as it occurs as a result of perturbed neural activity. It is conceivable that homeostatic plasticity has a protective role to reduce hyperactivity and mitigate depressive symptoms. Furthermore, the apical and basal dendrites showed different alterations in spine density and spine head volume at $24 \mathrm{~h}$ and $48 \mathrm{~h}$ post-stimulation. As the apical dendrites of pyramidal neurons in ACC preferentially connect to the contralateral ACC, prefrontal cortex, amygdala, and other brain areas (Fillinger et al., 2017), spinogenesis and spine head enlargement in the apical dendrites point towards enhanced communication between ACC and other regions during the progression of depressive disorder.

Our joint experimental-theoretical efforts provide evidence that structural plastic changes are homeostatically regulated, in parallel to functional changes. Our computational model of homeostatic structural plasticity was able to qualitatively predict the changes in network dynamics and connectivity triggered by external stimulation. 


\section{Supportive Information}

\section{Funding information}

This work was funded by the Universitätsklinikum Freiburg, University of Strasbourg, Centre National de la Recherche Scientifique (Grant/Award Number: UPR3212), NARSAD Young Investigator Grant from the Brain \& Behavior Research Foundation (Grant/Award Number: 24736) and Young Talent Award from the University of Strasbourg (IdEx award). Additional funding was obtained by BrainLinks-BrainTools (funded by the Federal Ministry of Economics, Science and Arts of Baden-Württemberg within the sustainability program for projects of the excellence initiative II) and by the Carl Zeiss Stiftung. We are grateful for support by the state of Baden-Württemberg through bwHPC and the German Research Foundation (DFG) through grant no INST 39/963-1 FUGG (bwForCluster NEMO). HL is funded by a NEUREX fellowship and the Deutsch-Französische Hochschule.

\section{Author contributions}

HL, JG, CN, SR, and IY conceived the project and designed the experiments. HL, JG, and SR established the computer model. HL performed the network simulation and analysis. HL performed all the mice experiments, collected data, and performed the analysis. CN and IY helped with the experiments. IY proposed the quantification strategy. HL wrote the manuscript, and all the authors revised and approved the paper.

\section{Acknowledgments}

We thank Elisabeth Waltisperger and Alessandro Bilella for advice on immunohistochemical staining. We thank Stephan Doridot and the Chronobiotron animal facilities for breeding and genotyping the mice. We thank Beyza Ayazgok for the efforts in molecular analysis. We thank Josef Bischofberger, Stefan Vestring, and Cyril Bories for their help setting up the microinjection experiments. We also thank the staff of the Life Imaging 
Center (LIC) in the Center for Biological Systems Analysis (ZBSA) of Freiburg University, in particular A. Naumann and R. Nitschke, for help with their confocal microscopy resources and their excellent support in image recording and analysis. Advice and code review by Martin Mörsdorf concerning the statistical analysis using linear mixed models are sincerely acknowledged. The authors also thank Sylvain Hugel, Rudi Tong, and Sandra Diaz-Pier for useful discussions. We also thank Uwe Grauer from the Bernstein Center Freiburg, as well as Bernd Wiebelt and Michael Janczyk from the Freiburg University Computing Center for their assistance with HPC applications and data storage. 


\section{References}

Barthas F, Humo M, Gilsbach R, Waltisperger E, Karatas M, Leman S, Hein L, Belzung C, Boutillier AL, Barrot M et al. (2017) Cingulate overexpression of mitogenactivated protein kinase phosphatase-1 as a key factor for depression. Biological Psychiatry $82: 370-379$.

Barthas F, Sellmeijer J, Hugel S, Waltisperger E, Barrot M, Yalcin I (2015) The anterior cingulate cortex is a critical hub for pain-induced depression. Biological Psychiatry $77: 236-245$.

Barton K (2019) MuMIn: Multi-Model Inference R package version 1.43.6.

Bates D, Mächler M, Bolker B, Walker S (2015) Fitting linear mixed-effects models using lme4. Journal of Statistical Software 67:1-48.

Becker N, Wierenga CJ, Fonseca R, Bonhoeffer T, Nägerl UV (2008) LTD induction causes morphological changes of presynaptic boutons and reduces their contacts with spines. Neuron 60:590-597.

Broadhead MJ, Horrocks MH, Zhu F, Muresan L, Benavides-Piccione R, DeFelipe J, Fricker D, Kopanitsa MV, Duncan RR, Klenerman D et al. (2016) PSD95 nanoclusters are postsynaptic building blocks in hippocampus circuits. Scientific Reports 6:1-14.

Brown CE, Aminoltejari K, Erb H, Winship IR, Murphy TH (2009) In vivo voltagesensitive dye imaging in adult mice reveals that somatosensory maps lost to stroke are replaced over weeks by new structural and functional circuits with prolonged modes of activation within both the peri-infarct zone and distant sites. Journal of Neuroscience 29:1719-1734.

Brunel N (2000) Dynamics of sparsely connected networks of excitatory and inhibitory spiking neurons. Journal of Computational Neuroscience 8:183-208. 
Bruun S, Stoeppler D, Keidel A, Kuhlmann U, Luck M, Diehl A, Geiger MA, Woodmansee D, Trauner D, Hegemann P et al. (2015) Light-dark adaptation of channelrhodopsin involves photoconversion between the all-trans and 13-cis retinal isomers. Biochemistry 54:5389-5400.

Butz M, van Ooyen A (2013) A simple rule for dendritic spine and axonal bouton formation can account for cortical reorganization after focal retinal lesions. PLOS Computational Biology 9.

Butz M, Woergoetter F, van Ooyen A (2009) Activity-dependent structural plasticity. Brain Research Reviews 60:287-305.

Cane M, Maco B, Knott G, Holtmaat A (2014) The relationship between PSD-95 clustering and spine stability in vivo. Journal of Neuroscience 34:2075-2086.

Cardin JA, Carlén M, Meletis K, Knoblich U, Zhang F, Deisseroth K, Tsai LH, Moore CI (2010) Targeted optogenetic stimulation and recording of neurons in vivo using celltype-specific expression of channelrhodopsin-2. Nature Protocols 5:247.

Caroni P, Donato F, Muller D (2012) Structural plasticity upon learning: regulation and functions. Nature Reviews Neuroscience 13:478-490.

Chen X, Nelson CD, Li X, Winters CA, Azzam R, Sousa AA, Leapman RD, Gainer H, Sheng M, Reese TS (2011) PSD-95 is required to sustain the molecular organization of the postsynaptic density. Journal of Neuroscience 31:6329-6338.

De Gois S, Schäfer MKH, Defamie N, Chen C, Ricci A, Weihe E, Varoqui H, Erickson JD (2005) Homeostatic scaling of vesicular glutamate and GABA transporter expression in rat neocortical circuits. Journal of Neuroscience 25:7121-7133.

Diaz-Pier S, Naveau M, Butz-Ostendorf M, Morrison A (2016) Automatic generation of connectivity for large-scale neuronal network models through structural plasticity. Frontiers in Neuroanatomy 10:57. 
Dissing-Olesen L, LeDue JM, Rungta RL, Hefendehl JK, Choi HB, MacVicar BA (2014) Activation of neuronal NMDA receptors triggers transient ATP-mediated microglial process outgrowth. Journal of Neuroscience 34:10511-10527.

Dumitriu D, Rodriguez A, Morrison JH (2011) High-throughput, detailed, cell-specific neuroanatomy of dendritic spines using microinjection and confocal microscopy. Nature Protocols 6:1391.

Ehrlich I, Klein M, Rumpel S, Malinow R (2007) PSD-95 is required for activity-driven synapse stabilization. Proceedings of the National Academy of Sciences 104:4176-4181.

Engert F, Bonhoeffer T (1999) Dendritic spine changes associated with hippocampal long-term synaptic plasticity. Nature 399:66-70.

Fauth M, Tetzlaff C (2016) Opposing effects of neuronal activity on structural plasticity. Frontiers in Neuroanatomy 10:75.

Fillinger C, Yalcin I, Barrot M, Veinante P (2017) Afferents to anterior cingulate areas 24a and 24b and midcingulate areas 24a' and 24b' in the mouse. Brain Structure and Function 222:1509-1532.

Franklin KB, Paxinos G et al. (2008) The mouse brain in stereotaxic coordinates Academic Press.

Fremeau RTJ, Voglmaier S, Seal RP, Edwards RH (2004) VGLUTs define subsets of excitatory neurons and suggest novel roles for glutamate. Trends in Neurosciences 27:98-103.

Gallinaro JV, Gasparovic N, Rotter S (2020) Homeostatic structural plasticity leads to the formation of memory engrams through synaptic rewiring in recurrent networks. bioRxiv .

Gallinaro JV, Rotter S (2018) Associative properties of structural plasticity based on firing rate homeostasis in recurrent neuronal networks. Scientific Reports 8:1-13. 
Garrido-García A, Andrés-Pans B, Durán-Trío L, Díez-Guerra FJ (2009) Activitydependent translocation of neurogranin to neuronal nuclei. Biochemical Journal 424:419-429.

Gipson CD, Olive MF (2017) Structural and functional plasticity of dendritic spines-root or result of behavior? Genes, Brain and Behavior 16:101-117.

Haydon PG, Nedergaard M (2015) How do astrocytes participate in neural plasticity? Cold Spring Harbor Perspectives in Biology 7:a020438.

Hengen KB, Pacheco AT, McGregor JN, Van Hooser SD, Turrigiano GG (2016) Neuronal firing rate homeostasis is inhibited by sleep and promoted by wake. Cell 165:180-191.

Hol EM, Pekny M (2015) Glial fibrillary acidic protein (GFAP) and the astrocyte intermediate filament system in diseases of the central nervous system. Current Opinion in Cell Biology 32:121-130.

Holtmaat A, Svoboda K (2009) Experience-dependent structural synaptic plasticity in the mammalian brain. Nature Reviews Neuroscience 10:647.

Huang KP, Huang FL, Shetty PK (2011) Stimulation-mediated translocation of calmodulin and neurogranin from soma to dendrites of mouse hippocampal CA1 pyramidal neurons. Neuroscience 178:1-12.

Humo M, Lu H, Yalcin I (2019) The molecular neurobiology of chronic pain-induced depression. Cell and tissue research pp. 1-23.

Jo S, Yarishkin O, Hwang YJ, Chun YE, Park M, Woo DH, Bae JY, Kim T, Lee J, Chun H et al. (2014) GABA from reactive astrocytes impairs memory in mouse models of alzheimer's disease. Nature Medicine 20:886.

Knott GW, Quairiaux C, Genoud C, Welker E (2002) Formation of dendritic spines with gabaergic synapses induced by whisker stimulation in adult mice. Neuron 34:265-273. 
Koga K, Descalzi G, Chen T, Ko HG, Lu J, Li S, Son J, Kim T, Kwak C, Huganir RL et al. (2015) Coexistence of two forms of LTP in ACC provides a synaptic mechanism for the interactions between anxiety and chronic pain. Neuron 85:377-389.

Lai CSW, Franke TF, Gan WB (2012) Opposite effects of fear conditioning and extinction on dendritic spine remodelling. Nature 483:87-91.

Lamprecht R, LeDoux J (2004) Structural plasticity and memory. Nature Reviews Neuroscience 5:45-54.

Letellier M, Levet F, Thoumine O, Goda Y (2019) Differential role of pre-and postsynaptic neurons in the activity-dependent control of synaptic strengths across dendrites. PLOS Biology 17:e2006223.

Liddelow SA, Barres BA (2017) Reactive astrocytes: production, function, and therapeutic potential. Immunity 46:957-967.

Linssen C, Lepperød ME, Mitchell J, Pronold J, Eppler JM, Keup C, Peyser A, Kunkel S, Weidel P, Nodem Y, Terhorst D, Deepu R, Deger M, Hahne J, Sinha A, Antonietti A, Schmidt M, Paz L, Garrido J, Ippen T, Riquelme L, Serenko A, Kühn T, Kitayama I, Mørk H, Spreizer S, Jordan J, Krishnan J, Senden M, Hagen E, Shusharin A, Vennemo SB, Rodarie D, Morrison A, Graber S, Schuecker J, Diaz S, Zajzon B, Plesser HE (2018) NEST 2.16.0.

Lisman J (2017) Glutamatergic synapses are structurally and biochemically complex because of multiple plasticity processes: long-term potentiation, long-term depression, short-term potentiation and scaling. Philosophical Transactions of the Royal Society B: Biological Sciences 372:20160260.

Lowel S, Singer W (1992) Selection of intrinsic horizontal connections in the visual cortex by correlated neuronal activity. Science 255:209-212. 
Lu H, Gallinaro JV, Rotter S (2019) Network remodeling induced by transcranial brain stimulation: A computational model of tdcs-triggered cell assembly formation. Network Neuroscience 3:924-943.

Ma Z, Turrigiano GG, Wessel R, Hengen KB (2019) Cortical circuit dynamics are homeostatically tuned to criticality in vivo. Neuron 104:655-664.

Matsuzaki M, Honkura N, Ellis-Davies GC, Kasai H (2004) Structural basis of long-term potentiation in single dendritic spines. Nature 429:761-766.

Nagel G, Szellas T, Huhn W, Kateriya S, Adeishvili N, Berthold P, Ollig D, Hegemann P, Bamberg E (2003) Channelrhodopsin-2, a directly light-gated cation-selective membrane channel. Proceedings of the National Academy of Sciences 100:13940-13945.

Nikolic K, Jarvis S, Grossman N, Schultz S (2013) Computational models of optogenetic tools for controlling neural circuits with light In 2013 35th Annual International Conference of the IEEE Engineering in Medicine and Biology Society (EMBC), pp. 5934-5937. IEEE.

Nollet M, Guisquet AML, Belzung C (2013) Models of depression: unpredictable chronic mild stress in mice. Current Protocols in Pharmacology 61:5-65.

Ohsawa K, Imai Y, Sasaki Y, Kohsaka S (2004) Microglia/macrophage-specific protein Iba1 binds to fimbrin and enhances its actin-bundling activity. Journal of Neurochemistry 88:844-856.

Oray S, Majewska A, Sur M (2004) Dendritic spine dynamics are regulated by monocular deprivation and extracellular matrix degradation. Neuron 44:1021-1030.

Pacheco AT, Tilden EI, Grutzner SM, Lane BJ, Wu Y, Hengen KB, Gjorgjieva J, Turrigiano GG (2019) Rapid and active stabilization of visual cortical firing rates across lightdark transitions. Proceedings of the National Academy of Sciences 116:18068-18077. 
Pfeiffer T, Poll S, Bancelin S, Angibaud J, Inavalli VK, Keppler K, Mittag M, Fuhrmann M, Nägerl UV (2018) Chronic 2P-STED imaging reveals high turnover of dendritic spines in the hippocampus in vivo. eLife 7:e34700.

Prichard L, Deloulme JC, Storm DR (1999) Interactions between neurogranin and calmodulin in vivo. Journal of Biological Chemistry 274:7689-7694.

R Core Team (2019) R: A Language and Environment for Statistical Computing R Foundation for Statistical Computing, Vienna, Austria.

Ramirez-Amaya V, Balderas I, Sandoval J, Escobar ML, Bermúdez-Rattoni F (2001) Spatial long-term memory is related to mossy fiber synaptogenesis. Journal of Neuroscience 21:7340-7348.

Reato D, Rahman A, Bikson M, Parra LC (2013) Effects of weak transcranial alternating current stimulation on brain activity - a review of known mechanisms from animal studies. Frontiers in Human Neuroscience 7:687.

Ryan TJ, Roy DS, Pignatelli M, Arons A, Tonegawa S (2015) Engram cells retain memory under retrograde amnesia. Science 348:1007-1013.

Samuels BA, Hen R (2011) Novelty-suppressed feeding in the mouse In Mood and anxiety related phenotypes in mice, pp. 107-121. Springer.

Sanderson TM, Georgiou J, Collingridge GL (2020) Illuminating relationships between the pre-and post-synapse. Frontiers in Neural Circuits 14.

Sellmeijer J, Mathis V, Hugel S, Li XH, Song Q, Chen QY, Barthas F, Lutz PE, Karatas M, Luthi A et al. (2018) Hyperactivity of anterior cingulate cortex areas 24a/24b drives chronic pain-induced anxiodepressive-like consequences. Journal of Neuroscience 38:3102-3115.

Sholl DA (1953) Dendritic organization in the neurons of the visual and motor cortices of the cat. Journal of Anatomy 87:387. 
Singec I, Knoth R, Ditter M, Volk B, Frotscher M (2004) Neurogranin is expressed by principal cells but not interneurons in the rodent and monkey neocortex and hippocampus. Journal of Comparative Neurology 479:30-42.

Tominaga-Yoshino K, Urakubo T, Okada M, Matsuda H, Ogura A (2008) Repetitive induction of late-phase LTP produces long-lasting synaptic enhancement accompanied by synaptogenesis in cultured hippocampal slices. Hippocampus 18:281-293.

Trachtenberg JT, Chen BE, Knott GW, Feng G, Sanes JR, Welker E, Svoboda K (2002) Long-term in vivo imaging of experience-dependent synaptic plasticity in adult cortex. Nature 420:788-794.

Turrigiano G (2012) Homeostatic synaptic plasticity: local and global mechanisms for stabilizing neuronal function. Cold Spring Harbor Perspectives in Biology 4:a005736.

Van Ooyen A (2011) Using theoretical models to analyse neural development. Nature Reviews Neuroscience 12:311-326.

Weinhard L, di Bartolomei G, Bolasco G, Machado P, Schieber NL, Neniskyte U, Exiga M, Vadisiute A, Raggioli A, Schertel A et al. (2018) Microglia remodel synapses by presynaptic trogocytosis and spine head filopodia induction. Nature Communications 9:1228.

Williams JC, Xu J, Lu Z, Klimas A, Chen X, Ambrosi CM, Cohen IS, Entcheva E (2013) Computational optogenetics: empirically-derived voltage-and light-sensitive channelrhodopsin-2 model. PLOS Computational Biology 9.

Wilson NR, Kang J, Hueske EV, Leung T, Varoqui H, Murnick JG, Erickson JD, Liu G (2005) Presynaptic regulation of quantal size by the vesicular glutamate transporter vglut1. Journal of Neuroscience 25:6221-6234.

Xia Z, Storm DR (2005) The role of calmodulin as a signal integrator for synaptic plasticity. Nature Reviews Neuroscience 6:267-276.

Yizhar O, Fenno LE, Davidson TJ, Mogri M, Deisseroth K (2011) Optogenetics in neural systems. Neuron 71:9-34. 
Zamani A, Sakuragi S, Ishizuka T, Yawo H (2017) Kinetic characteristics of chimeric channelrhodopsins implicate the molecular identity involved in desensitization. Biophysics and Physicobiology 14:13-22.

Zamanian JL, Xu L, Foo LC, Nouri N, Zhou L, Giffard RG, Barres BA (2012) Genomic analysis of reactive astrogliosis. Journal of Neuroscience 32:6391-6410.

Zhang J, Zhang D, McQuade JS, Behbehani M, Tsien JZ, Xu M (2002) C-fos regulates neuronal excitability and survival. Nature Genetics 30:416-420.

Zhang W, Vazquez L, Apperson M, Kennedy MB (1999) Citron binds to PSD-95 at glutamatergic synapses on inhibitory neurons in the hippocampus. Journal of Neuroscience 19:96-108.

Zhong L, Gerges NZ (2012) Neurogranin targets calmodulin and lowers the threshold for the induction of long-term potentiation. PLOS ONE 7.

Zhou Q, Homma KJ, Poo Mm (2004) Shrinkage of dendritic spines associated with long-term depression of hippocampal synapses. Neuron 44:749-757.

Zuur A, Ieno EN, Walker N, Saveliev AA, Smith GM (2009) Mixed effects models and extensions in ecology with $R$ Springer Science \& Business Media. 


\section{Legends}

Figure 1: The HSP model and predictions of the current study. A The linear growth rule of axonal boutons (empty triangles) and dendritic spines (dark red ticks). B Temporal evolution of neural activity and network connectivity in response to optogenetic stimulation. Light blue shaded areas label the stimulation period. Blue and dark gray curves in the upper panel represent the firing rate of stimulated and non-stimulated neurons. Blue, dark gray, and light gray curves in the lower panel represent the intra-group connectivity of the stimulated population, intra-group connectivity of the non-stimulated population, and the inter-group connectivity between two populations. C Diagram of synapse reorganization. The blue area covers the stimulated neurons. Blue and dark gray synapses are from the stimulated and non-stimulated neurons to a stimulated neuron. D The connection matrix of all excitatory neurons at three discrete time points labeled by orange dots in panel $\mathbf{B}$. The $\mathrm{x}$ and $\mathrm{y}$ ticks are the pre- and postsynaptic neuron IDs. The color indicates the average connection probability. White square labels the intra-group connectivity of the stimulated neurons. E Repetitive optogenetic stimulation boosted the connectivity among the stimulated neurons. Small insets display the dynamics within $48 \mathrm{~h}$ after the last stimulation session. A and $\mathbf{C}$ were adapted from our previous work $\mathrm{Lu}$ et al. (2019).

Figure 2: Consecutive optogenetic stimulation triggered hyperactivity in ACC and depressive-like behavior in mice. A-C Experimental design of the current study. D-F Mice used for immunohistochemical staining experiments showed depressive-like behavior at $24 \mathrm{~h}$ and $48 \mathrm{~h}$ post-stimulation. D Splash test results of mice sacrificed at $24 \mathrm{~h}$ post-stimulation ( $p=0.003$, Mann Whitney $\mathrm{U}$ test; $N=9$ for sham and $N=10$ for stimulated). E-F Results of NSF and splash tests for mice sacrificed at $48 \mathrm{~h}$ ( $p=0.01$ and $p=0.005$, Mann Whitney $\mathrm{U}$ test; $N=5$ for sham and $N=5$ for stimulated mice). G c-Fos expression was elevated by the optogenetic stimulation at $1.5 \mathrm{~h}(p<0.001)$ and specifically on the ipsilateral side $(p<0.01)$, decayed to baseline at $24 \mathrm{~h}$ and $48 \mathrm{~h}$ post-stimulation. The $\mathrm{x}$ ticks label the mean distance of stained sections (-, anterior; 
+ , posterior) from the optical fiber. The y axis labels the c-Fos + cell number within the masks of stained sections. GLMM was used for the statistical analysis. $p<0.05$, $p<0.01$, and $p<0.001$ means $95 \%$ confidence interval (CI), 99\% CI, and $99.9 \%$ CI does not cross zero respectively. Data showed in the left panel was from viral transfection mice (Extended Data Figure 2-1).

Figure 3: Neuronal dendritic tree structure was not drastically affected by the optogenetic stimulation at $24 \mathrm{~h}$ and $48 \mathrm{~h}$. A Mice sacrificed at $24 \mathrm{~h}$ and $48 \mathrm{~h}$ for microinjection experiments showed depressive-like behavior in splash and NSF tests (for both batches, $N=6$ for sham and $N=6$ for the stimulated; $p=0.006, p=0.012$, and $p=0.004$ respectively, Mann Whitney U test). B Representative example of neurons filled with red fluorescent dye. C Overall distribution of pyramidal neurons injected in layer 2-3 of ACC from both hemispheres for both batches. Black dots are from sham mice, and red dots are from the stimulated mice. For the 24 h-post group, we selected 32 well-injected neurons in total and 15 neurons were from sham mice; each mouse contributed 2.67 neurons on average $(\mathrm{SD}=1.31)$. For the $48 \mathrm{~h}$-post group, we selected 24 neurons in total and 12 neurons were from sham mice; each mouse contributed 2 neurons on average $(\mathrm{SD}=2.12)$. D The soma size was not changed by stimulation (LMM). E Dendritic tree structure was not altered by stimulation (GLMM).

Figure 4: Spine density and spine head volume showed the opposite changes at $24 \mathrm{~h}$ and $48 \mathrm{~h}$ post-stimulation. A-B Representative example of filled dendritic segments. C-D Spine density at $24 \mathrm{~h}$ (84 dendritic segments from sham and 111 segments from stimulated mice) and $48 \mathrm{~h}$ (101 segments from sham and 61 segments from stimulated mice). E-F Spine density of each class. G-H Cumulative distribution of spine head volume. LMM was used for statistical analysis.

Figure 5: Expression of VGLUT1 and PSD-95 were upregulated by optogenetic stimulation at $24 \mathrm{~h}$ and decayed at $48 \mathrm{~h}$ post-stimulation. A, C Representative images of VGLUT1 and PSD-95 staining on the ipsilateral hemisphere of sections within $0.1 \mathrm{~mm}$ anterior-posterior (AP) to the optic fiber from both sham and stimulated mice. B VGLUT1 fluorescent intensity at different time and distance to the fiber optic. At $1.5 \mathrm{~h}$, 
the main effects of optogenetic stimulation (sham/stimulated) was not significant. At $24 \mathrm{~h}$, the main effect of optogenetic stimulation was significant $(p<0.001)$ and its interaction with the distance to the fiber optic and side of the stimulation were significant $($ sham $/$ stimulated $\times$ distance, $p<0.001 ;$ sham/stimulated $\times$ ipsi/contra, $p<0.001)$. At $48 \mathrm{~h}$, no significant difference was detected. D The PSD-95 fluorescent intensity at different time and distance. At $1.5 \mathrm{~h}$, the main effects of optogenetic stimulation was not significant. At $24 \mathrm{~h}$, the main effect of stimulation was significant $(p<0.01)$ and its interaction with the distance to the fiber optic and side of the stimulation were significant (sham/stimulated $\times$ distance, $p<0.001 ;$ sham $/$ stimulated $\times$ ipsi/contra, $p<0.001)$. At $48 \mathrm{~h}$, main effects of stimulation is significant $(p<0.01)$. LMM was used for statistical analysis.

Figure 6: Optogenetic stimulation failed to alter the expression of neurogranin, but triggered its cellular translocation. A Representative images of neurogranin staining on the ipsilateral hemisphere of sections within $0.1 \mathrm{~mm}$ anterior-posterior to the optic fiber from both sham and stimulated mice. White arrowheads indicate the soma of pyramidal neurons. B In terms of fluorescent intensity, no significant effects were observed between stimulated and sham mice (LMM). C Representative images of neurogranin distribution in soma and region around soma. White solid and dashed lines indicate the soma or the equivalent dendrites respectively. D The scatter plots of soma area and its relative intensity of neurogranin at $1.5 \mathrm{~h}, 24 \mathrm{~h}$, and $48 \mathrm{~h}$. The relative intensity in the neural soma for sham mice was all above 1 . The relative intensity of neural soma from stimulated mice was slightly increased at $1.5 \mathrm{~h}$, greatly dropped below 1 at $24 \mathrm{~h}$, and recovered to a level lower than sham at $48 \mathrm{~h}(p<0.001, p<0.001, p<0.001$ respectively, Mann Whitney U test).

Figure 7: Expression of GFAP and IBA1 were upregulated by optogenetic stimulation at $24 \mathrm{~h}$ and $48 \mathrm{~h}$ post-stimulation. A, C Representative images of GFAP and IBA1 staining on the ipsilateral hemisphere of sections within $0.1 \mathrm{~mm}$ AP to the optic fiber from both sham and the stimulated mice. B The GFAP fluorescent intensity at different time and distance. At $1.5 \mathrm{~h}$, the main effects of optogenetic stimulation, side of stimulation, 
and distance to the optic fiber were significant $(p<0.01, p<0.001$, and $p<0.001$ respectively). At $24 \mathrm{~h}$, the main effects of optogenetic stimulation, side of stimulation, and distance to the optic fiber were again significant $(p<0.001, p<0.001$, and $p<0.001$ respectively); their interaction effects were also significant (sham/stimulated $\times$ distance, $p<0.01 ;$ sham/stimulated $\times$ ipsi/contra, $p<0.001)$. At $48 \mathrm{~h}$, the main effects of stimulation, side of stimulation, and distance to the optic fiber were significant $(p<0.001$, $p<0.001, p<0.001)$. LMM was used for statistical analysis. D The IBA1+ cell counting at different time and distance to the optic fiber. At $1.5 \mathrm{~h}$, the main effects of side of stimulation and distance to the optic fiber were significant $(p<0.001$ and $p<0.001)$. At $24 \mathrm{~h}$, the main effects of optogenetic stimulation, side of stimulation, and distance to the optic fiber were significant $(p<0.001, p<0.001, p<0.001)$; their interaction effects were also significant (sham/stimulated $\times$ distance, $p<0.01$; sham/stimulated $\times$ ipsi/contra, $p<0.001$ ). At $48 \mathrm{~h}$, the main effects of optogenetic stimulation was significant $(p<0.001)$. GLMM was used for statistical analysis.

Figure 8: Summary of the computational model and mouse experimental results. Light blue shaded areas indicate four optogenetic stimulation sessions ( $\mathrm{S} 1$ to $\mathrm{S} 4,30 \mathrm{~min}$ per session). Red and blue curves represent the model-predicted temporal evolution of neural activity and connectivity of stimulated neurons Colored symbols in the right panel represent the respectively expression level of c-Fos, synaptic proteins, and glial markers at $1.5 \mathrm{~h}, 24 \mathrm{~h}$, and $48 \mathrm{~h}$ post-stimulation in the stimulated mice relative to its corresponding sham group. Neurogranin presented here is its relative expression in the dendrites. The gray dashed line is the baseline. Symbols above the baseline denote that the measurements of the stimulated mice is increased than sham and vice versa. The illustration of neuron with dendrites and spines shows the spine density and spine head volume were increased at $24 \mathrm{~h}$ but decreased at $48 \mathrm{~h}$ post-stimulation. 


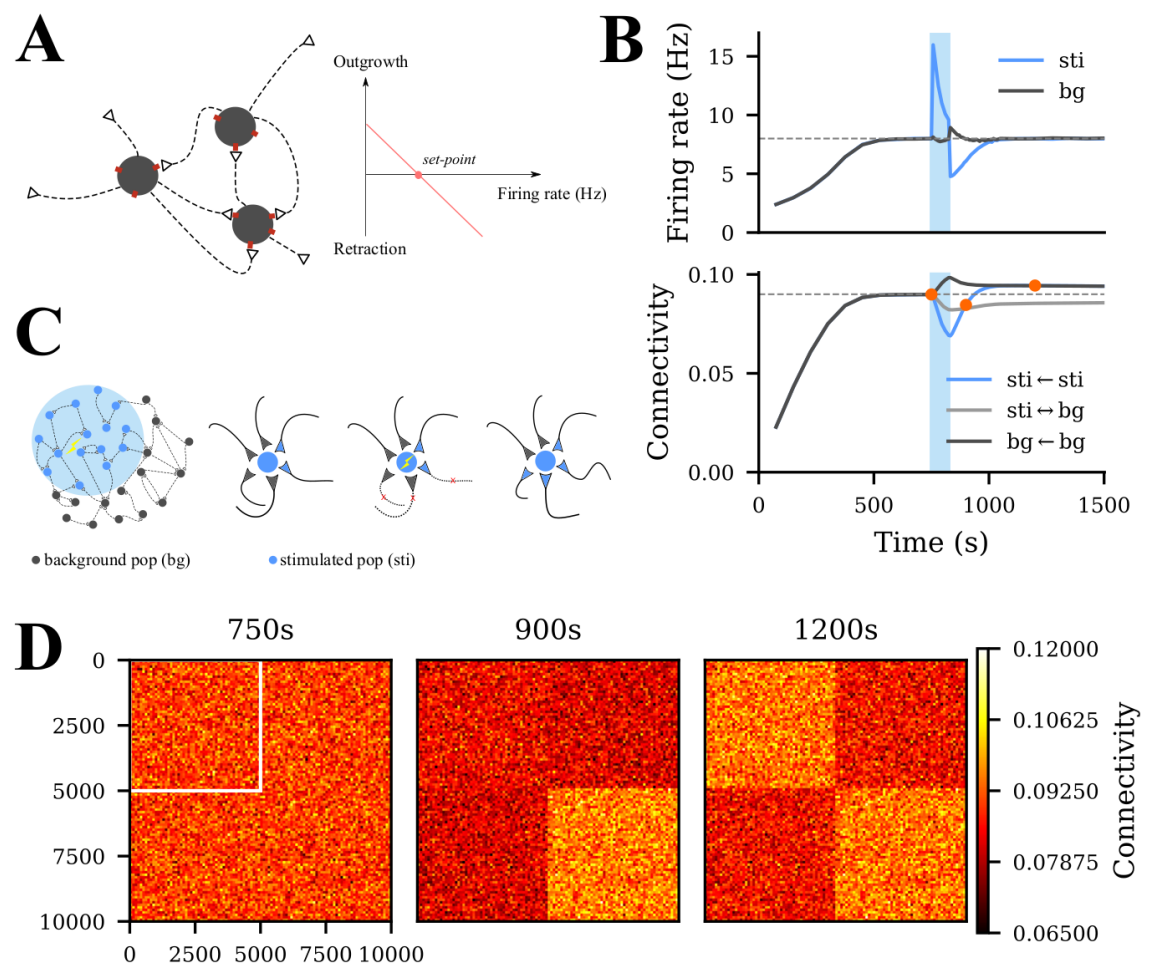

$\mathbf{E}$
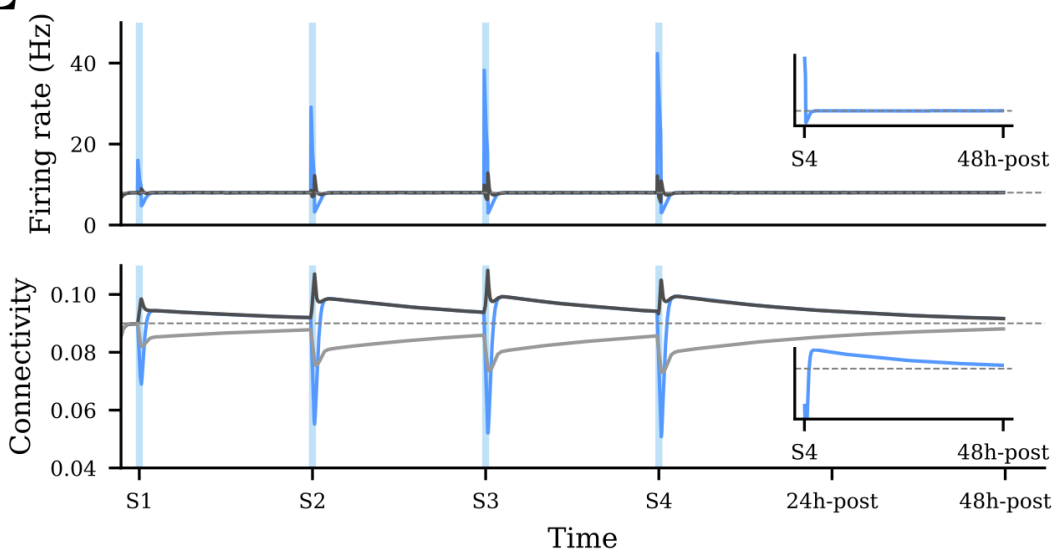

Figure 1 
A

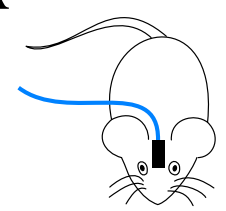

Thy1-ChR2-YFP

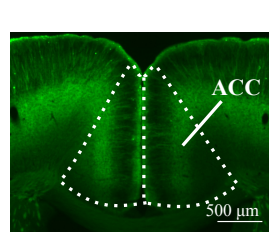

$20 \mathrm{~Hz}, 30 \mathrm{~min}$
C

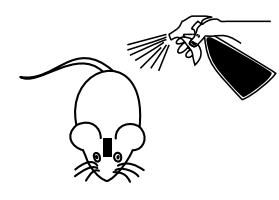

Splash test
D

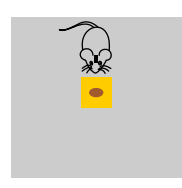

NSF test
B
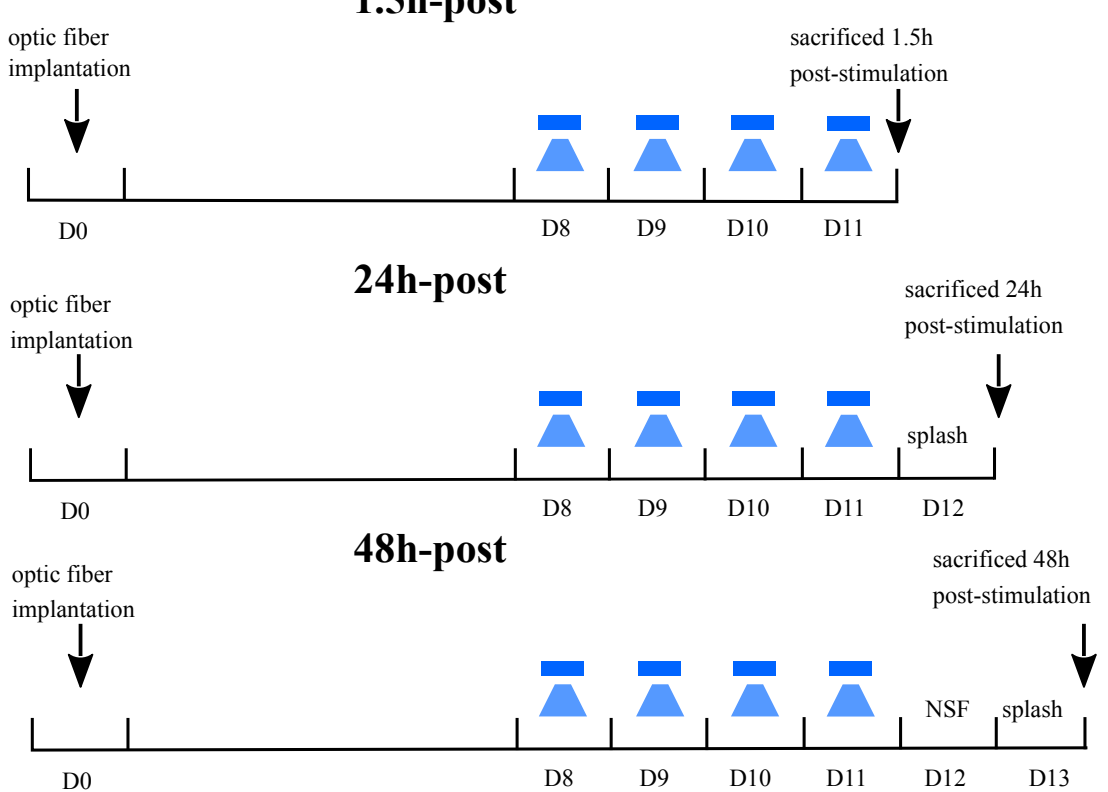

G

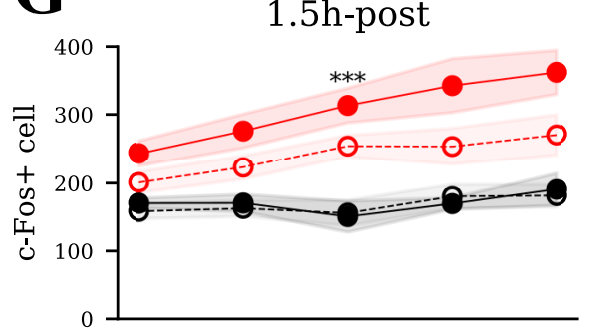

24h-post
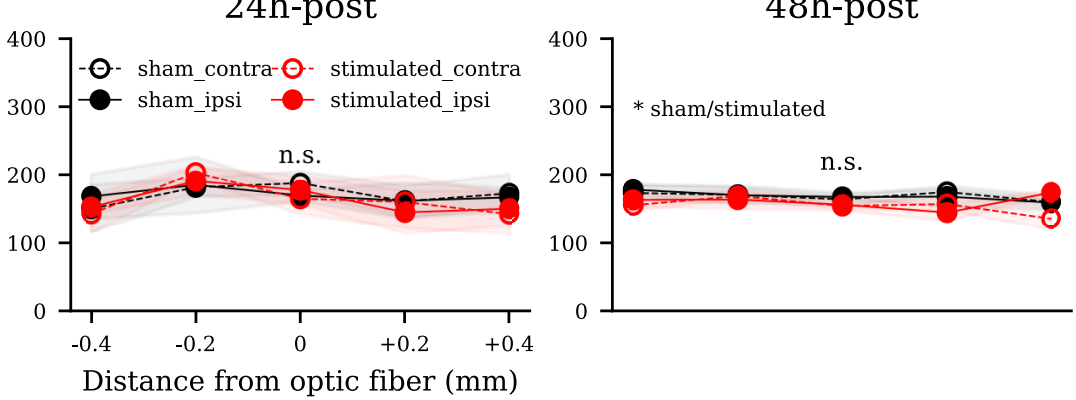

Figure 2 
bioRxiv preprint doi: https://doi org/10.1101/2020.09.16.297606; this version posted September 17, 2020. The copyright holder for this preprint (which was not certified by peer review) is the author/funder, who has granted bioRxiv a license to display the preprint in perpetuity. It is made available under aCC-BY-NC-ND 4.0 International license.

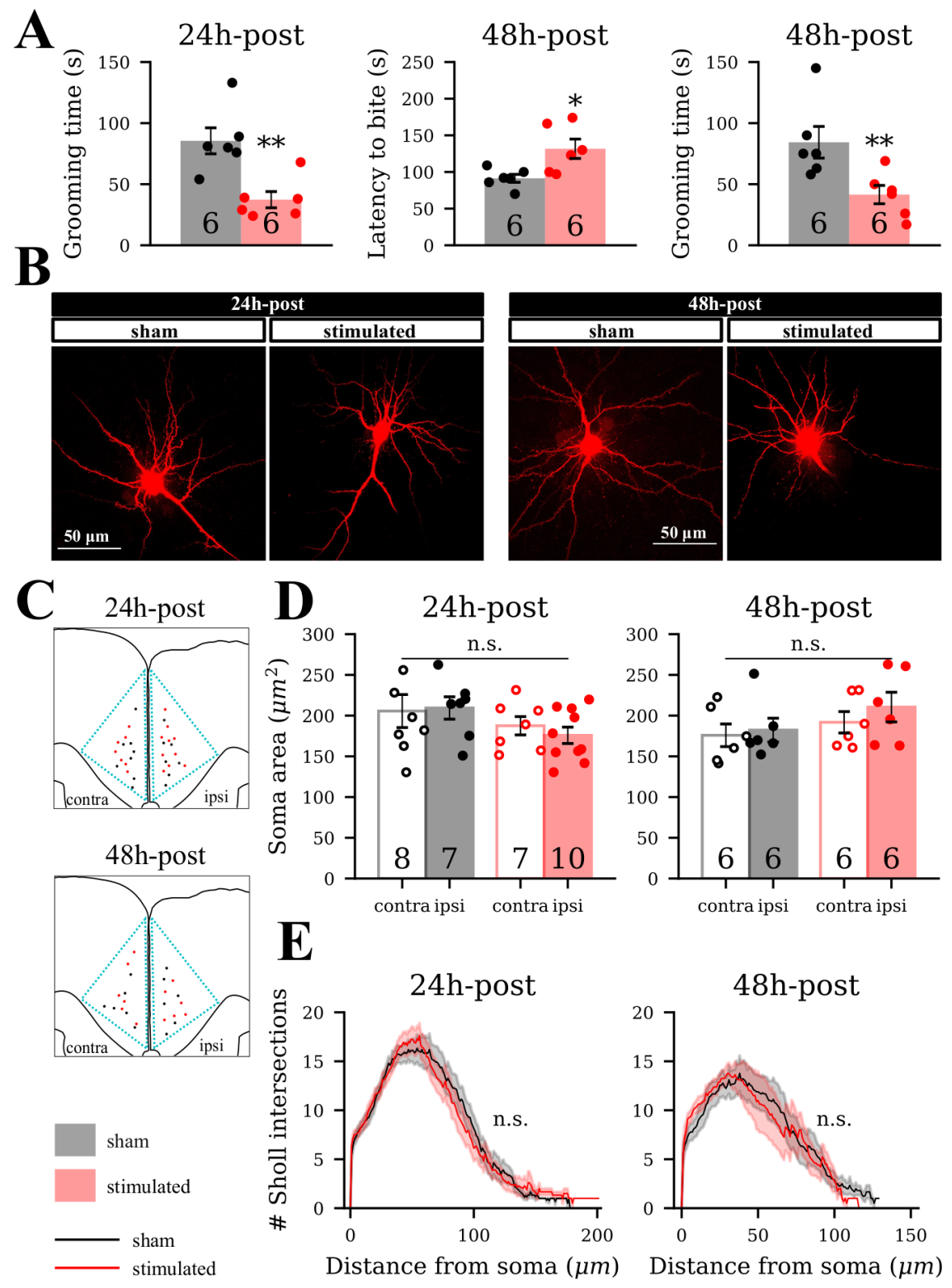

Figure 3 


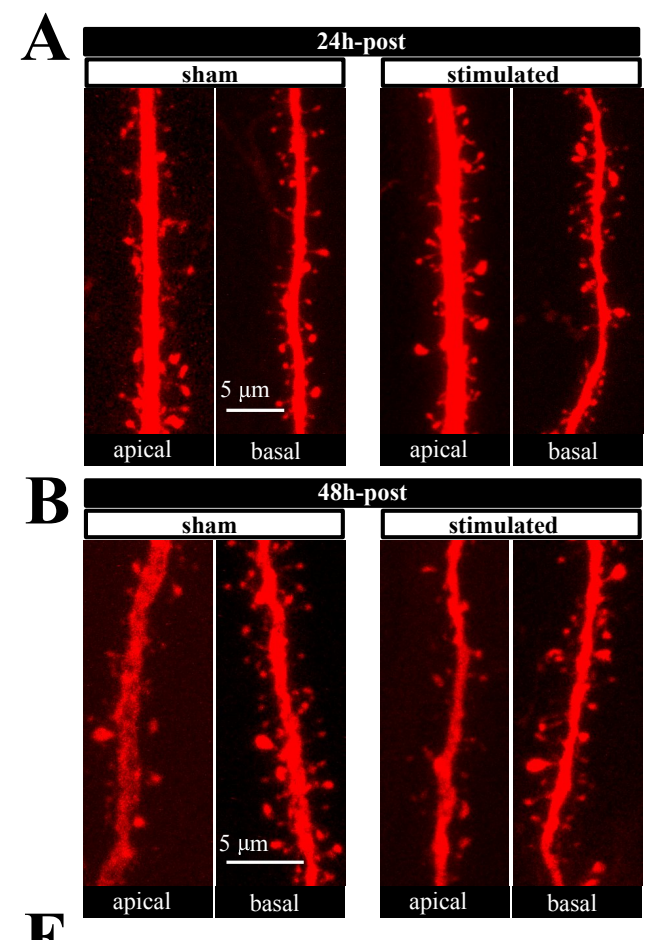

$\mathbf{E}$
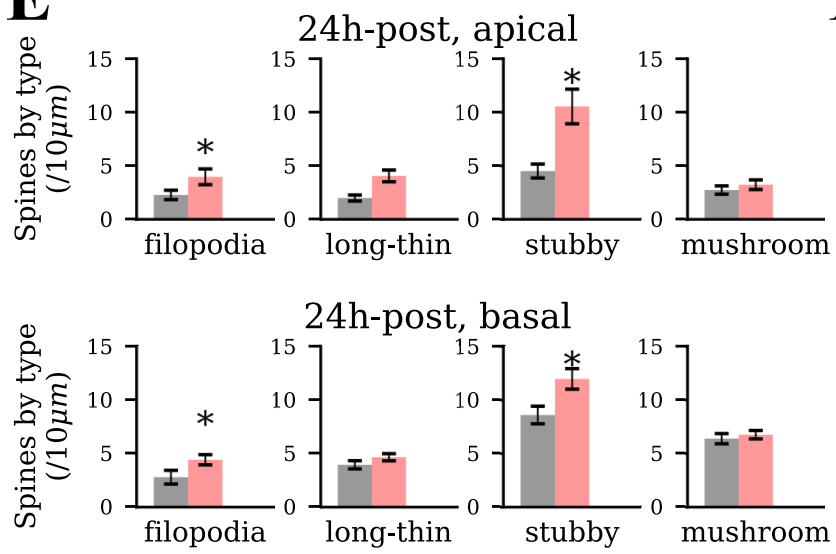

$\mathbf{G}$

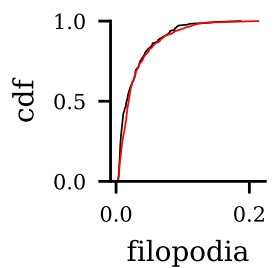

24h-post, apical
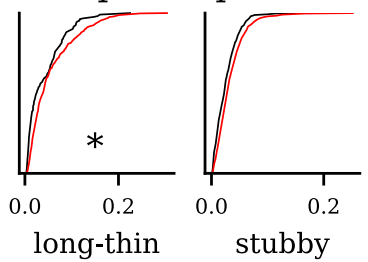

24h-post, basal
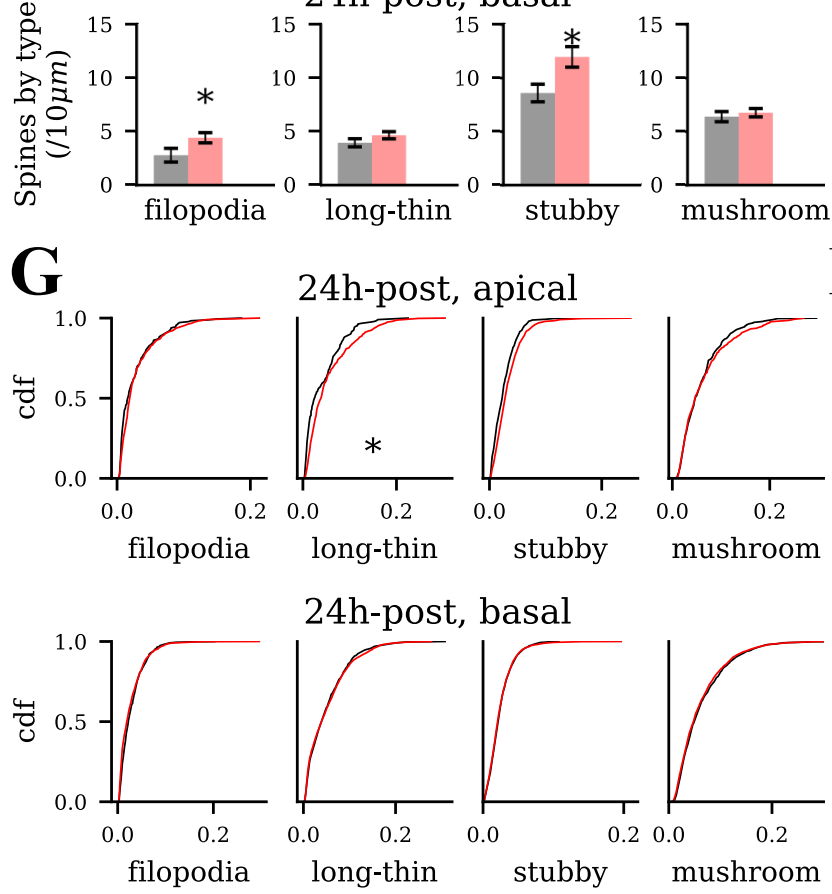
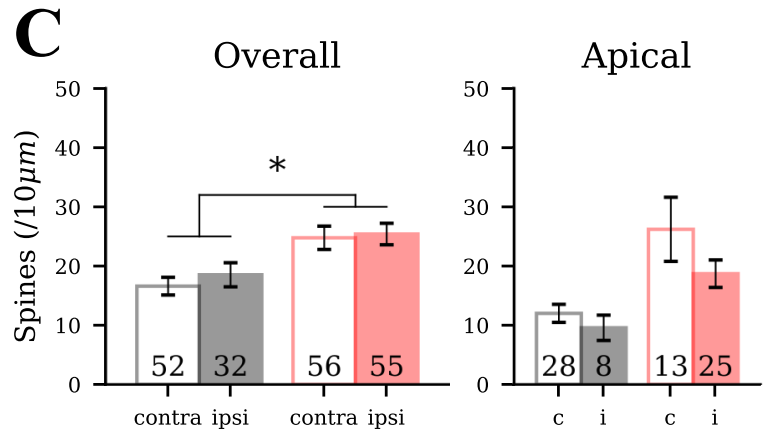

Apical

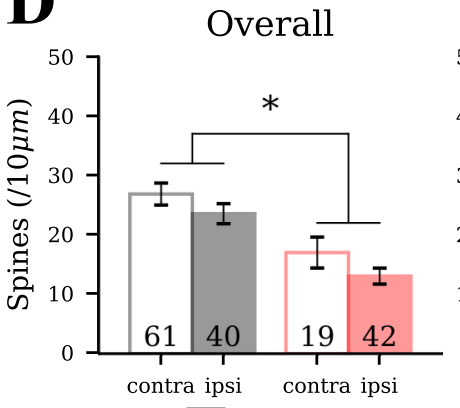

$\mathbf{F}$

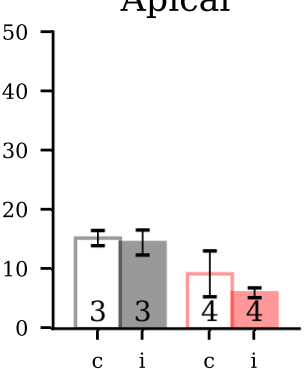

48h-post, apical
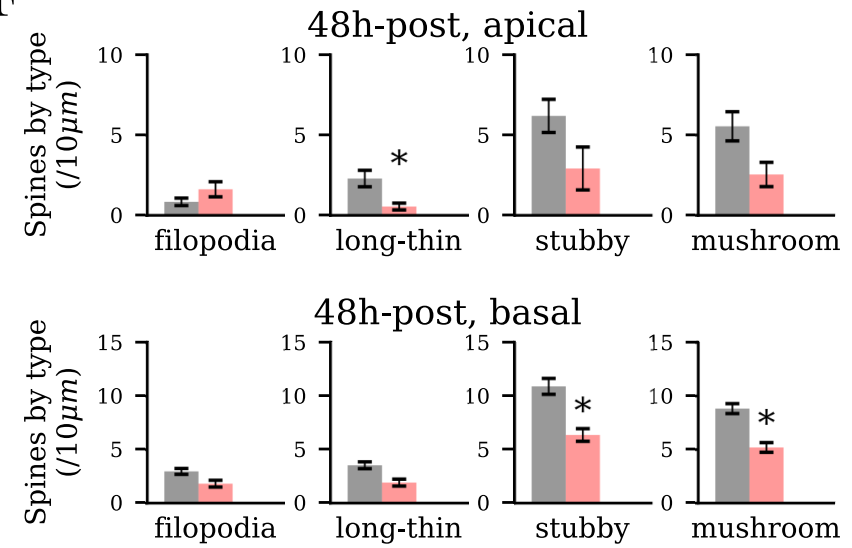

48h-post, basal
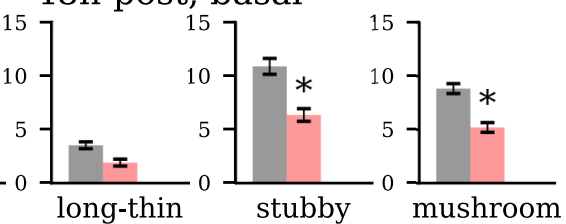

H

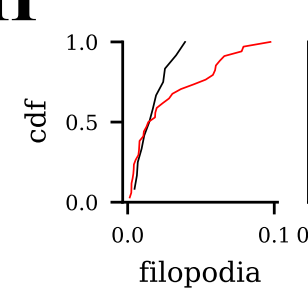

48h-post, apical
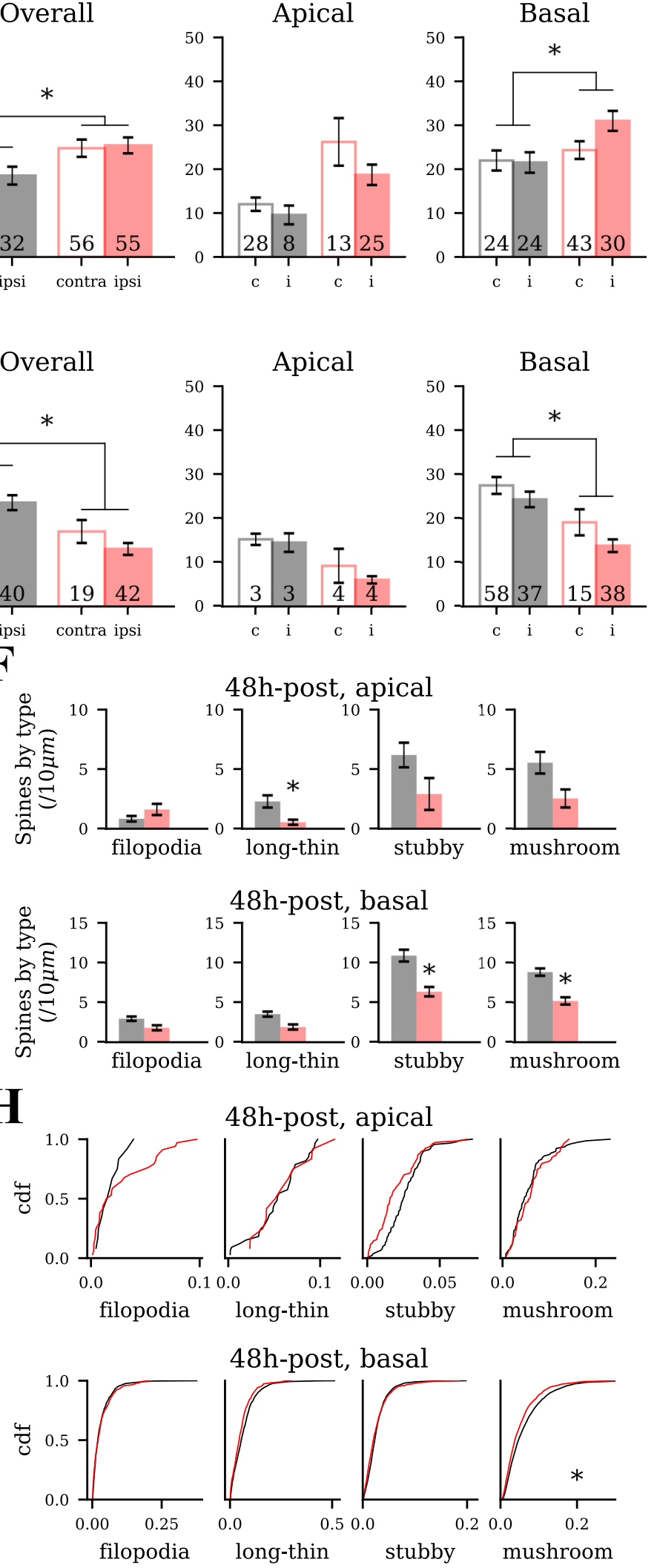

48h-post, basal

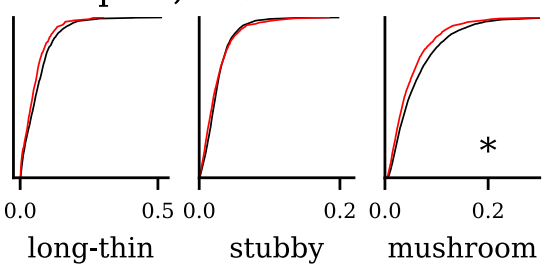

Figure 4 
bioRxiv preprint doi: https://doi.org/10.1101/2020.09.16.297606; this version posted September 17, 2020. The copyright holder for this

preprint (which was not certified by peer review) is the author/funder, who has granted bioRxiv a license to display the preprint in perpetuity. It is made available under aCC-BY-NC-ND 4.0 International license.

A

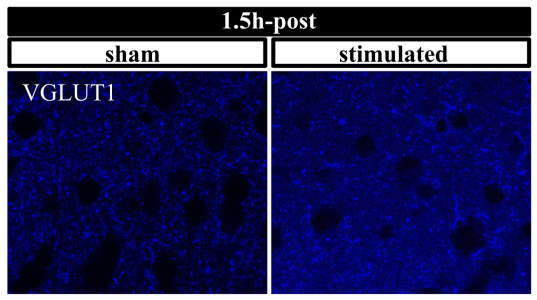

B

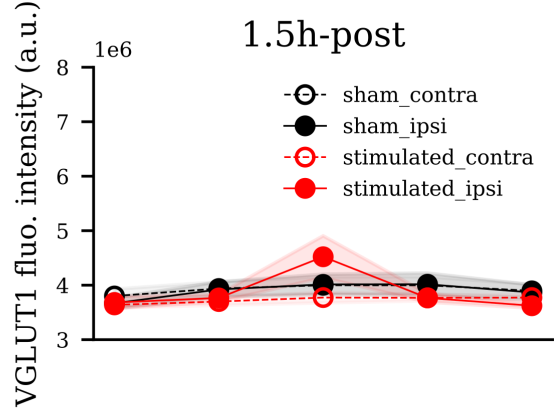

C

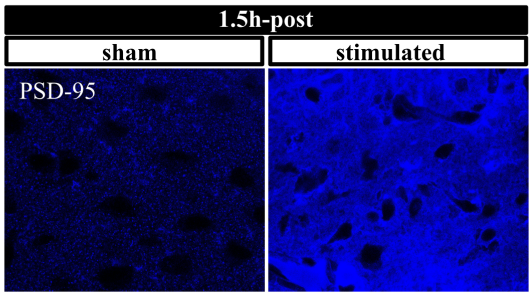

D

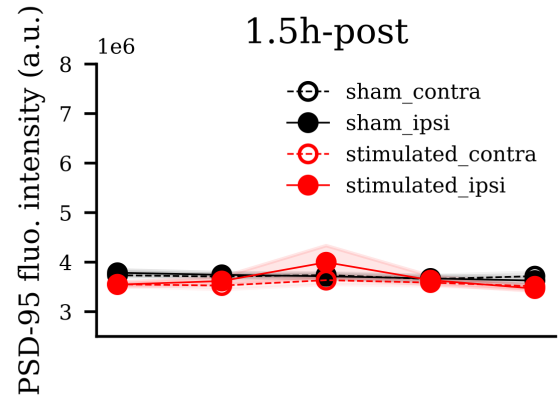

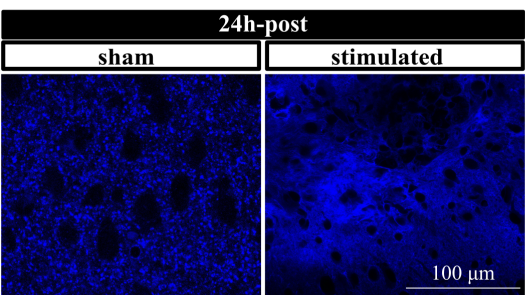
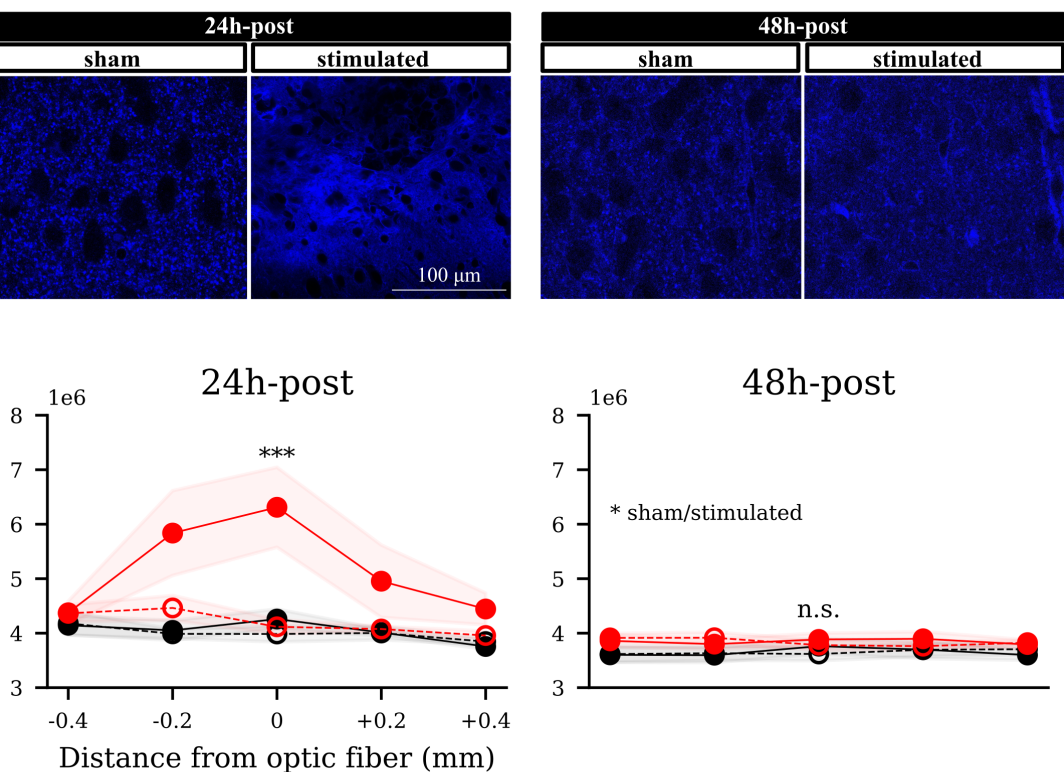

Distance from optic fiber $(\mathrm{mm})$
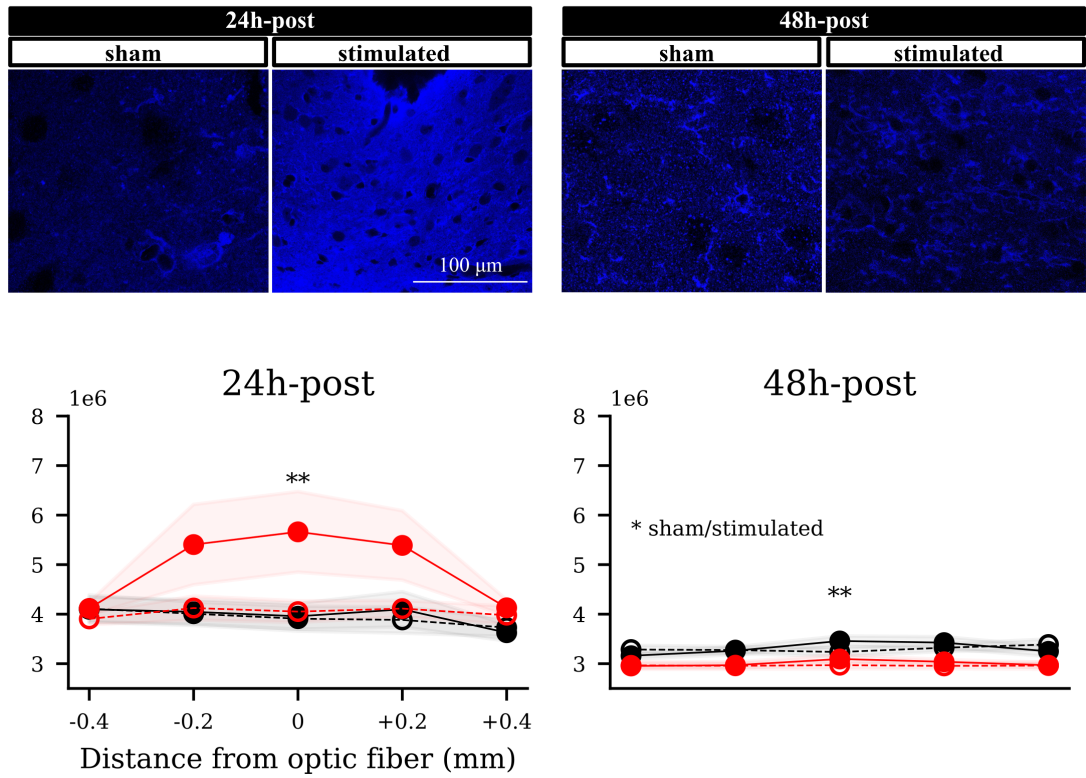

Figure 5 
bioRxiv preprint doi: https://doi. org/10.1101/2020.09.16.297606: this version posted September 17 2020. The copyright holder for this

preprint (which was not certified by peer review) is the author/funder, who has granted bioRxiv a license to display the preprint in perpetuity. It is made available under aCC-BY-NC-ND 4.0 International license.

A

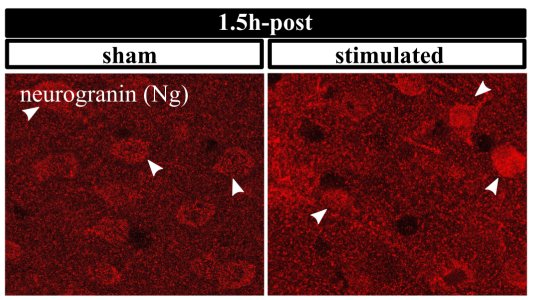

B

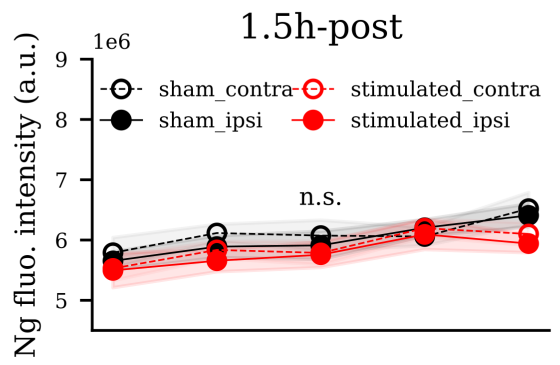

C

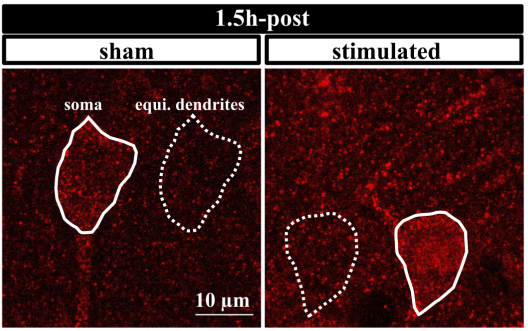

D

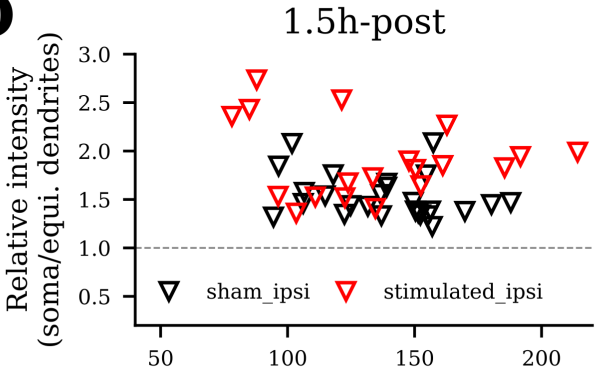

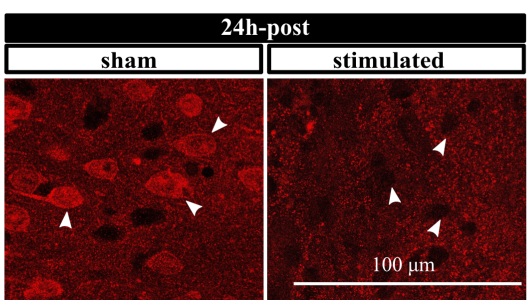

24h-post
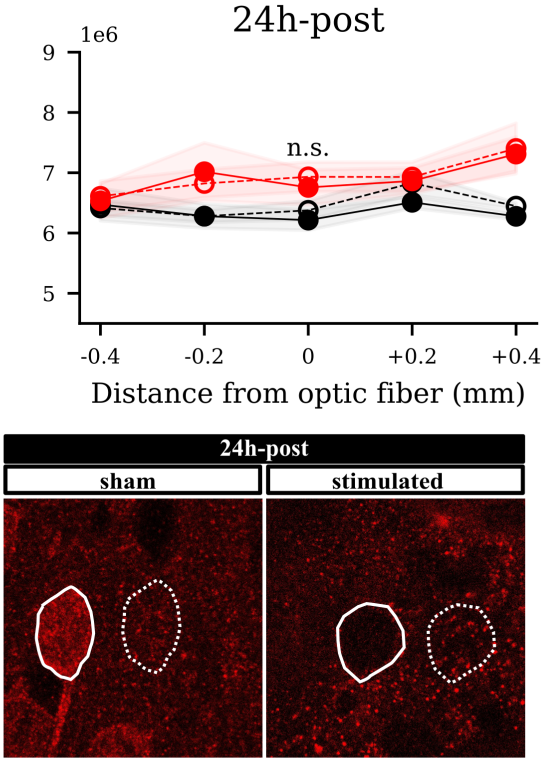

24h-post
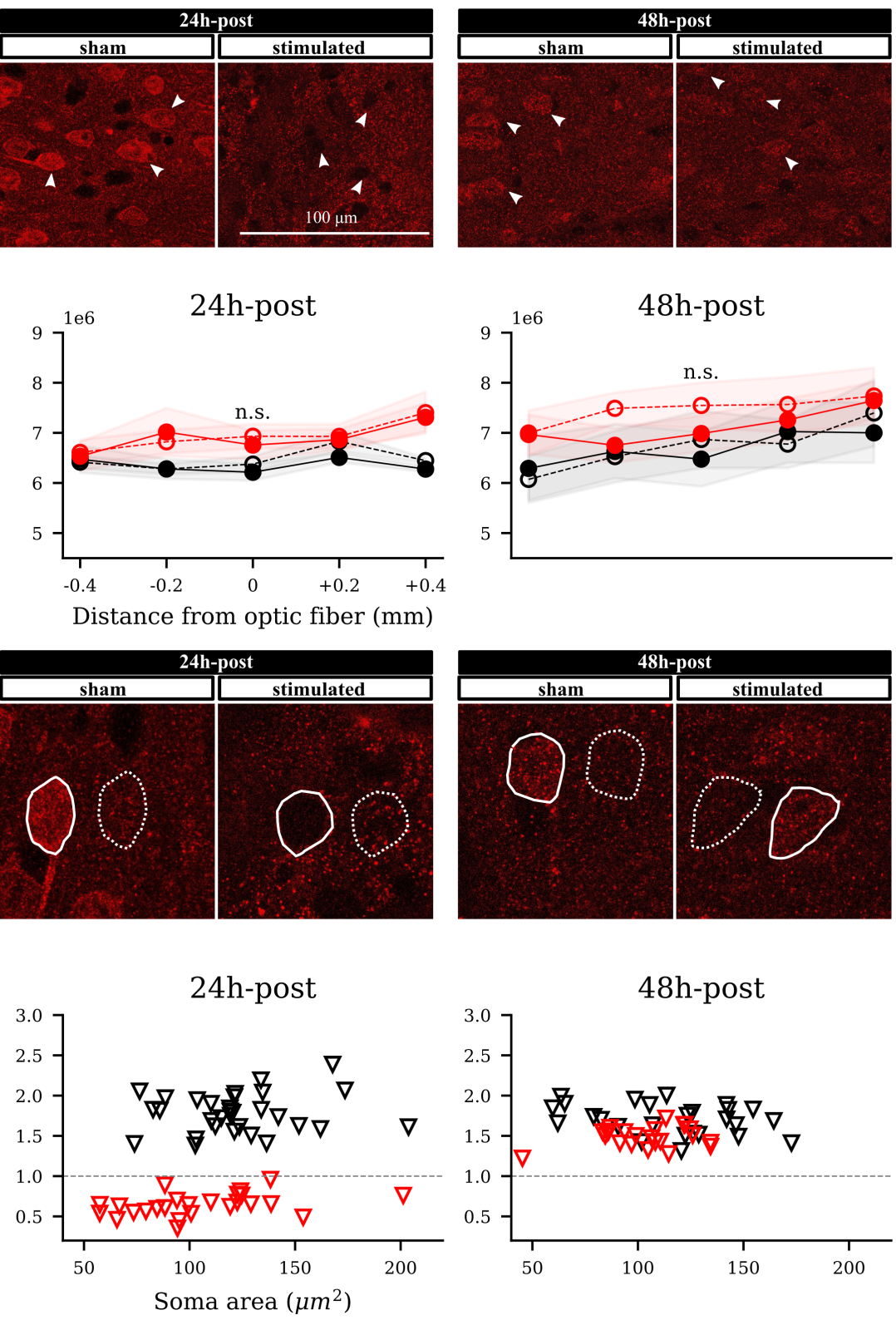

48h-post
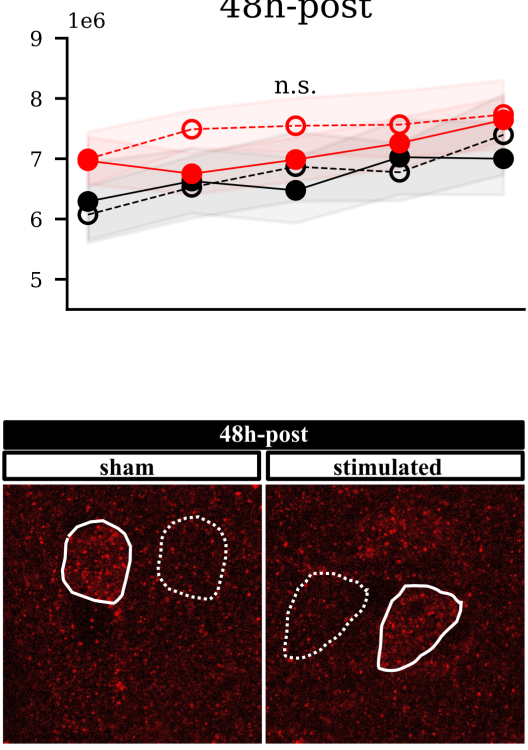

48h-post

Figure 6 
bioRxiv preprint doi: https://doi.org/10.1101/2020.09.16.297606: this version posted September 17, 2020. The copyright holder for this

preprint (which was not certified by peer review) is the author/funder, who has granted bioRxiv a license to display the preprint in perpetuity. It is made available under aCC-BY-NC-ND 4.0 International license.

A

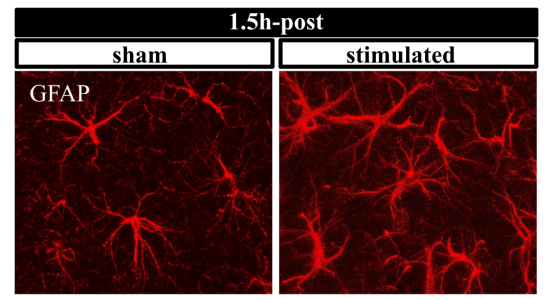

B

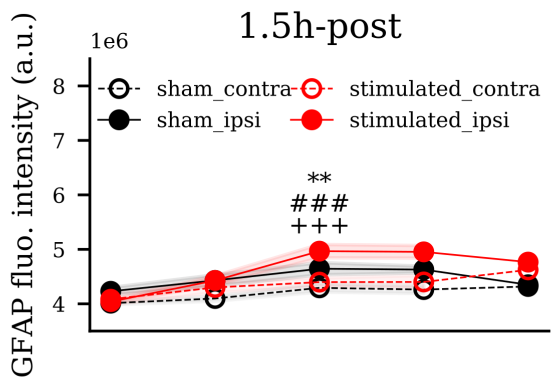

C

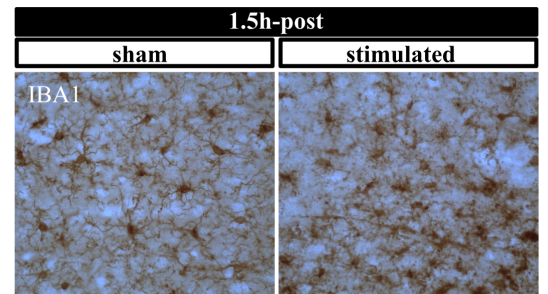

D

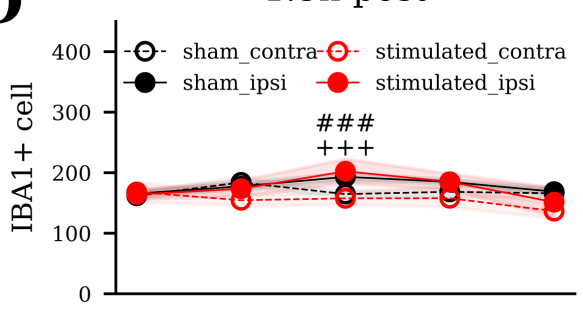

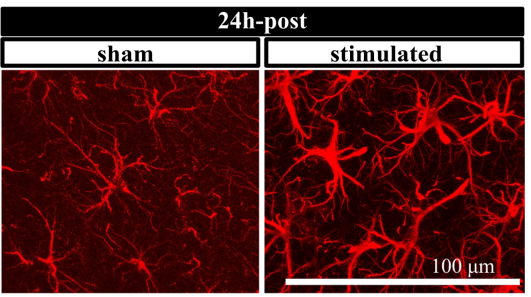
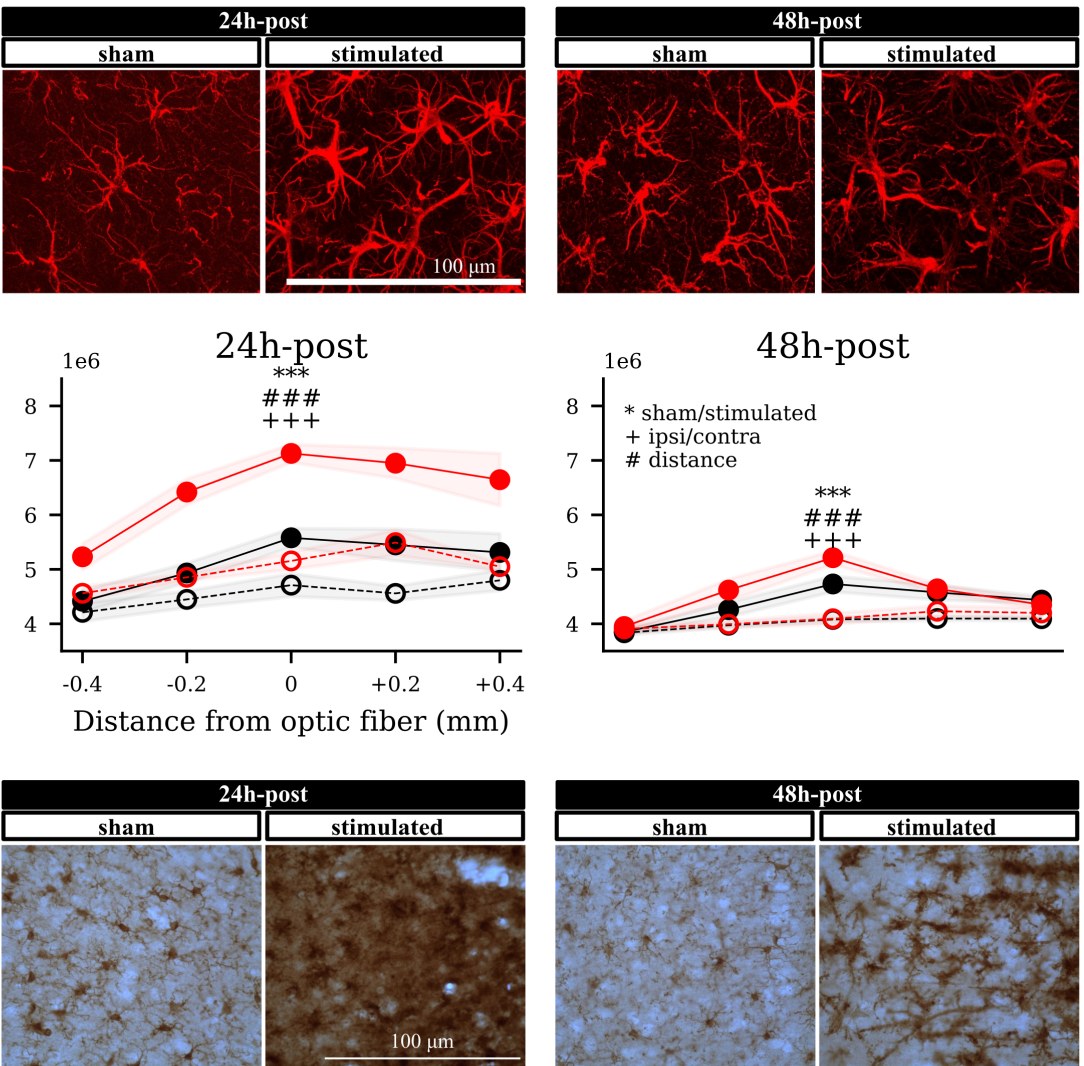

24h-post

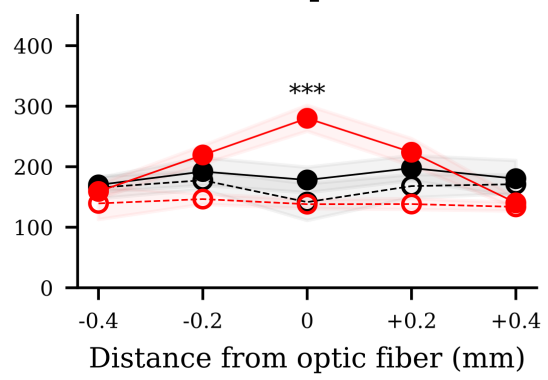

48h-post

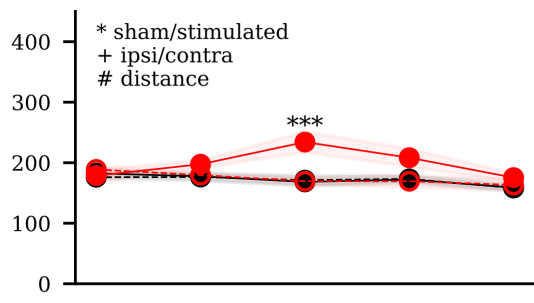

Figure 7 

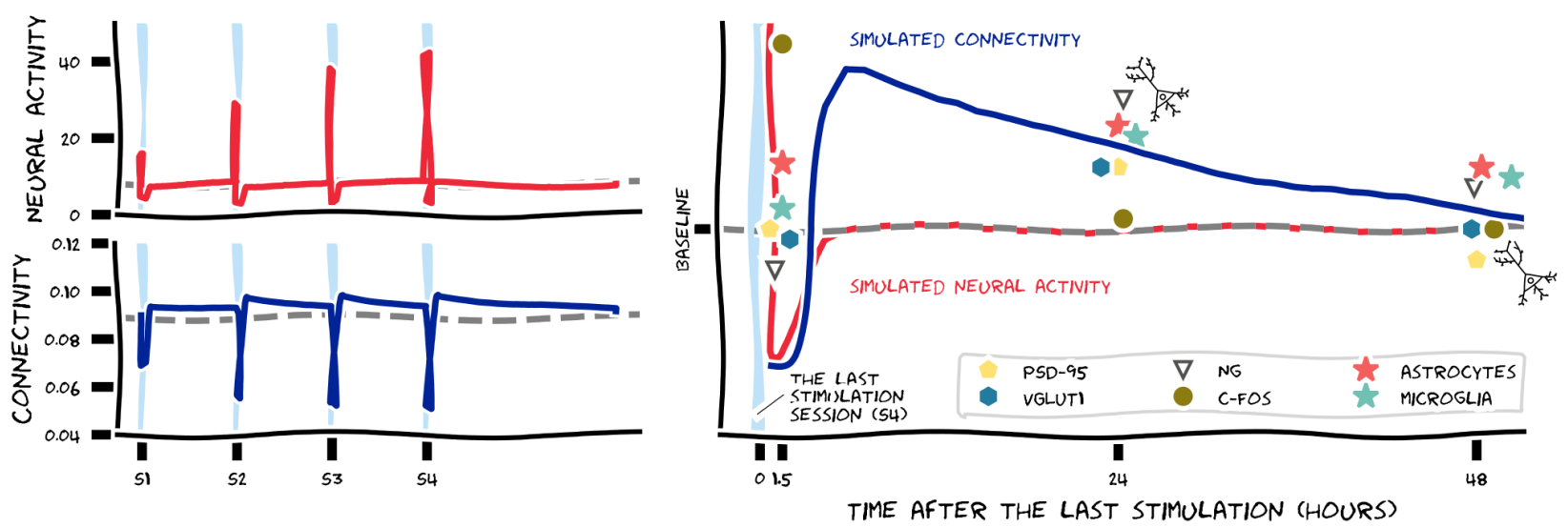

Figure 8 